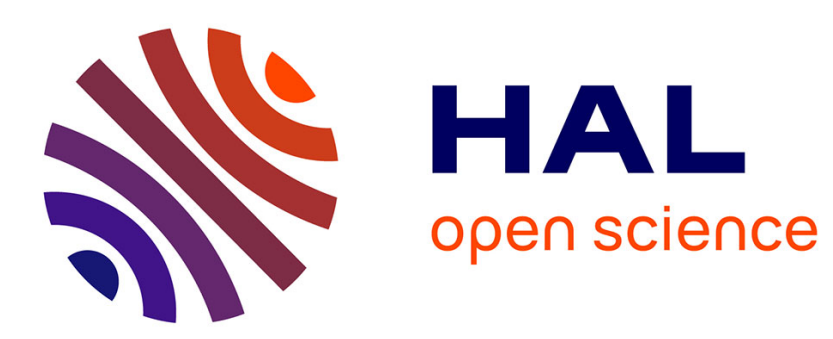

\title{
Asymptotic expansions for interior penalty solutions of control constrained linear-quadratic problems
}

Felipe Alvarez, Jérôme Bolte, J. Frederic Bonnans, Francisco Silva

\section{To cite this version:}

Felipe Alvarez, Jérôme Bolte, J. Frederic Bonnans, Francisco Silva. Asymptotic expansions for interior penalty solutions of control constrained linear-quadratic problems. Mathematical Programming, Series A, 2012, 135 (1-2), pp.473-507. inria-00365540

\section{HAL Id: inria-00365540 \\ https://hal.inria.fr/inria-00365540}

Submitted on 3 Mar 2009

HAL is a multi-disciplinary open access archive for the deposit and dissemination of scientific research documents, whether they are published or not. The documents may come from teaching and research institutions in France or abroad, or from public or private research centers.
L'archive ouverte pluridisciplinaire HAL, est destinée au dépôt et à la diffusion de documents scientifiques de niveau recherche, publiés ou non, émanant des établissements d'enseignement et de recherche français ou étrangers, des laboratoires publics ou privés. 
INSTITUT NATIONAL DE RECHERCHE EN INFORMATIQUE ET EN AUTOMATIQUE

\section{Asymptotic expansions for interior penalty solutions of control constrained linear-quadratic problems}

Felipe Álvarez — Jérôme Bolte — J. Frédéric Bonnans — Francisco J. Silva

$\mathbf{N}^{\circ} 6863$

Mars 2008

Thème NUM

apport

de recherche 



\title{
Asymptotic expansions for interior penalty solutions of control constrained linear-quadratic problems
}

\author{
Felipe Álvarez* , Jérôme Bolte ${ }^{\dagger}$, J. Frédéric Bonnans ${ }^{\ddagger}$, Francisco \\ J. Silva ${ }^{\S}$ \\ Thème NUM — Systèmes numériques \\ Équipes-Projets Commands \\ Rapport de recherche $n^{\circ} 6863$ - Mars 2008 - 26 pages
}

\begin{abstract}
We consider a quadratic optimal control problem governed by a nonautonomous affine differential equation subject to nonnegativity control constraints. For a general class of interior penalty functions, we show how to compute the principal term of the pointwise expansion of the state and the adjoint state. Our main argument relies on the following fact: If the control of the initial problem satisfies strict complementarity conditions for its Hamiltonian except for a finite number of times, the estimates for the penalized optimal control problem can be derived from the estimations of a related stationary problem.

Our results provide several types of efficiency measures of the penalization technique: error estimations of the control for $L^{s}$ norms $(s$ in $[1,+\infty])$, error estimations of the state and the adjoint state in Sobolev spaces $W^{1, s}$ ( $s$ in $[1,+\infty))$ and also error estimates for the value function.

For the $L^{1}$ norm and the logarithmic penalty, the optimal results are given. In this case we indeed establish that the penalized control and the value function errors are of order $O(\varepsilon|\log \varepsilon|)$.
\end{abstract}

Key-words: Optimal control, interior-point algorithms, sensitivity, expansion of value function and solutions.

\footnotetext{
* Department of Mathematical Engineering, Universidad de Chile and Centre for Mathematical Modelling, Universidad de Chile-CNRS. Casilla 170-3 Santiago 3, Chile. This author was partially supported by grants from FONDECYT 1050706 and the Millennium Scientific Institute on Complex Engineering Systems funded by MIDEPLAN-Chile (falvarez@dim.uchile.cl)

$\dagger$ Université Paris 06, Equipe Combinatoire et Optimisation, UMR 7090, Case 189, 75252 Paris, France and INRIA-Saclay and CMAP, École Polytechnique, 91128 Palaiseau, France (bolte@math.jussieu.fr)

¥ INRIA-Saclay and CMAP, École Polytechnique, 91128 Palaiseau, France (Frederic.Bonnans@inria.fr)

$\S$ INRIA-Saclay and CMAP, École Polytechnique, 91128 Palaiseau, France (silva@cmap.polytechnique.fr)
}

\author{
Centre de recherche INRIA Saclay - Île-de-France \\ Parc Orsay Université \\ 4, rue Jacques Monod, 91893 ORSAY Cedex \\ Téléphone : +331 72925900
}




\section{Développements asymptotiques pour les solutions intérieures de pénalité d'un problème linéaire quadratique avec contraintes sur la commande}

Résumé : On considère un problème de commande optimale dont la fonction coût est quadratique et la dynamique est régie par une équation différentielle ordinaire. Pour une classe générale de fonctions de pénalité intérieure, on montre comment calculer le terme principal du développement ponctuelle de l'état et de l'état adjoint. Notre argument principal se base sur le fait suivant: Si la commande optimale pour le problème initial satisfait les conditions de complémentarité stricte pour son Hamiltonien sauf en un nombre fini d'instants, les estimations pour le problème de commande optimale pénalisé peuvent être obtenues à partir des estimations pour un problème stationnaire associé. Nos résultats fournissent plusieurs types de mesures d'efficacité pour la technique de pénalisation: estimations des erreurs de la commande pour les normes $L^{s}$ $(s$ dans $[1,+\infty])$, estimations des erreurs pour l'état et l'état adjoint dans les espaces de Sobolev $W^{1, s}(s$ dans $[1,+\infty))$ et aussi des estimations des erreurs pour la fonction valeur.

Pour la norme $L^{1}$ et la pénalisation logarithmique, on montre que les erreurs pour la commande optimale du problème pénalise et pour la fonction valeur sont de l'ordre $O(\varepsilon|\log \varepsilon|)$.

Mots-clés : Commande optimale, algorithmes de point intérieur, sensibilité, développement de la valeur et des solutions. 


\section{Introduction}

For finite dimensional optimization problems interior-point methods are recognized as being presently among the most efficient algorithms. For detailed expositions of the theory and recent developments see, for instance, $[9,13,20]$ and references therein. In particular, path-following algorithms based on the logarithmic penalty are very popular by their well-known convergence properties (see [6] Part IV and [10]).

Penalty and interior-point methods are especially well-suited for optimal control problems. A possible procedure is indeed as follows: fix a small penalty parameter, write the optimality conditions of the resulting unconstrained problem, discretize the system and apply a procedure for solving nonlinear equations. This discretization can be analyzed and evaluated with a good precision, allowing to design efficient grid refinement algorithms $[2,8]$. On the other hand the system of equations corresponding to optimality conditions has a Jacobian with a band structure and can be, for instance, efficiently solved using QR factorization algorithm (see [2]). The corresponding approach has been applied to real-world aerospace optimization problems (see [3]).

When the dynamics are described by an ordinary differential equation, interiorpoint methods have been investigated by several authors (see e.g. [11, 12, 16, 19]). Some convergence results are discussed in [7] and [16]. The latter uses a primal-dual interior point method, based in the Fisher-Burmeister complementarity function, obtains $O(\sqrt{\varepsilon})$ error estimation for the $L^{\infty}$ norm and linear convergence of a short-step path-following method.

For the PDE framework see $[4,5,14,17,18]$. In [17] a control reduced method is developed and error estimates of $O(\sqrt{\varepsilon})$ for the $L^{\infty}$ norm are obtained. Superlinear convergence is established in [14] . See also [15] for a $L^{s}$-analysis $(s \in[2,+\infty[)$ where global linear and local superlinear convergence are studied.

In this work we consider a rather general linear-quadratic optimal control problem where the dynamics are described by a nonautonomous affine differential equation, while nonnegativity restrictions are imposed on the control. For this kind of problems the theoretical result obtained in [16] is not applicable (at least because of the non-boundedness of the constraint set). Our approach is complementary to that of [16] in which a continuation method is used to show the convergence of the central path. Instead, we start from the optimal solution and obtain the existence of the central path in the "spirit" of the classical implicit function theorem. Our central result relies indeed on a standard restoration result (see Theorem 20) whose applicability depends on the strict uniform derivability of the family of "optimality mappings" induced by the penalized problems. We prove that this last differentiability property can be deduced from the following simple geometrical assumption: as time elapses the control of the initial problem satisfies strict complementarity conditions with respect to its Hamiltonian (except perhaps for a finite number of times). Within this framework error estimations of the state, adjoint state, control and value functions are derived from some associated stationary problems. These estimations depend on the regularity of the underlying dynamics: they involve either $L^{s}$ norms or Sobolev norms (see Theorem 13).

In the particular case of the logarithmic penalty, one recovers the $O(\sqrt{\varepsilon})$ bound for the control error in the $L^{\infty}$ norm and a bound of order $O(\varepsilon|\log \varepsilon|)$ for the $L^{1}$ norm. This last fact is deduced from a transversality assumption and 
implies an important improvement of the error estimation of the cost function (see Theorem 17). This estimate is optimal in view of the example solved in [1].

On the other hand, asymptotic expansions of state and the adjoint state are obtained. This result together with the strict complementarity assumptions provide a deeper understanding of the interplay between the variations of the optimal control and its junction points (times where the set of active constraints changes). In addition our results are of a general nature, and deal with a general interior penalty.

The paper is organized as follows: Section 2 is devoted to the problem statement and the description of its penalized versions; standard results revolving around these aspects are recalled. In Section 3 some associated stationary problems are described into depth, this allows in Section 4 to establish our main results. The last Section provides illustrative applications and a throughout study of the logarithmic penalty case for which optimal bounds are provided.

The proof of the restoration result which is an important tool of the present paper was provided in [1], its proof is reproduced in the Appendix.

\section{Problem statement and preliminary results}

For $m \in \mathbb{N}$ denote respectively by $\mathbb{R}_{+}^{m}, \mathbb{R}_{++}^{m}$ the subsets of $\mathbb{R}^{m}$ of nonnegative coordinates and positive coordinates. For every $x \in \mathbb{R}^{m}$ we will write $x^{i}$ for its $i$-th coordinate and $|\cdot|$ for its Euclidean norm. Define $\mathcal{U}:=L^{2}\left([0, T] ; \mathbb{R}^{m}\right)$ and $\mathcal{U}_{+}:=L^{2}\left([0, T] ; \mathbb{R}_{+}^{m}\right)$. Given $n \in \mathbb{N}$ and $s \in[1, \infty]$ set $L^{s}:=L^{s}\left([0, T] ; \mathbb{R}^{n}\right)$, and define the Sobolev space by

$$
W^{1, s}:=\left\{y \in L^{s}\left([0, T] ; \mathbb{R}^{n}\right) ; \dot{y} \in L^{s}\left([0, T] ; \mathbb{R}^{n}\right)\right\},
$$

where $\dot{y}$ is the derivative of $y$ in the distribution sense $\left(^{1}\right)$.

The standard norms of these spaces are denoted by $\|\cdot\|_{s}$ and $\|\cdot\|_{1, s}$ respectively. For $m \in \mathbb{N}$, denote respectively by $\mathcal{S}^{m}, \mathcal{S}_{+}^{m}, \mathcal{S}_{++}^{m}$ the set of symmetric, symmetric positive semidefinite, symmetric positive definite matrices of order $m$. For $S \in \mathcal{S}_{++}^{m}$, let $\lambda_{\min }(S)$ (resp. $\left.\lambda_{\max }(S)\right)$ denote the smallest (resp. largest) eigenvalue of $S$.

Let $m, n$ be two positive integers. Consider the following state equation

$$
\dot{y}_{t}=A_{t} y_{t}+B_{t} u_{t}+\psi_{t}, \quad t \in(0, T) ; \quad y_{0}=x_{0},
$$

with data $T>0, A \in \mathcal{C}^{0}\left([0, T] ; \mathbb{R}^{n \times n}\right), B \in \mathcal{C}^{0}\left([0, T] ; \mathbb{R}^{n \times m}\right), x_{0} \in \mathbb{R}^{n}, \psi \in$ $L^{1}\left([0, T] ; \mathbb{R}^{n}\right)$. For any control $u \in \mathcal{U},(1)$ has a unique solution in $W^{1,1}$ denoted by $y[u]$ and called the state associated with $u$.

It is well known that the mapping $u \mapsto y[u]$ is linear continuous from $\mathcal{U}$ into $W^{1,1}$. Let $R \in \mathcal{C}^{0}\left([0, T] ; \mathcal{S}_{++}^{m}\right), C \in \mathcal{C}^{0}\left([0, T] ; \mathcal{S}_{+}^{n}\right), \varphi \in L^{1}$, and $M \in \mathcal{S}_{++}^{m}$. Consider the application $g$ defined by

$$
(u, y, t) \in \mathbb{R}^{m} \times \mathbb{R}^{n} \times[0, T] \mapsto g(u, y, t):=\frac{1}{2} u^{\top} R_{t} u+\frac{1}{2} y^{\top} C_{t} y+\varphi_{t}^{\top} y,
$$

and the cost function $J_{0}: \mathcal{U} \rightarrow \mathbb{R}$ defined by

$$
J_{0}(u):=\int_{0}^{T} g\left(u_{t}, y[u](t)-\bar{y}_{t}, t\right) \mathrm{d} t+\frac{1}{2} y[u](T)^{\top} M y[u](T),
$$

\footnotetext{
${ }^{1}$ As usual each element of $W^{1, s}$ will systematically be represented by a continuous function.
} 
where $\bar{y} \in \mathcal{C}^{2}\left([0, T], \mathbb{R}^{n}\right)$ is a reference state function.

Let us consider the following linear-quadratic optimal control problem:

$$
\text { Min } J_{0}(u) \text { subject to }(1) \text { and } u \in \mathcal{U}_{+} \text {. }
$$

Since $\mathcal{U}_{+}$is a closed subset of $\mathcal{U}$, the continuous and strongly convex function $J_{0}$ has a unique minimum $u_{0}$ over $\mathcal{U}_{+}$. For $(u, y, p, t) \in \mathbb{R}_{+}^{m} \times \mathbb{R}^{n} \times \mathbb{R}^{n} \times[0, T]$, the classical Hamiltonian for $\left(\mathcal{C} \mathcal{P}_{0}\right)$ is defined by

$$
H_{0}(u, y, p, t):=\frac{1}{2} u^{\top} R_{t} u+\frac{1}{2}\left(y-\bar{y}_{t}\right)^{\top} C_{t}\left(y-\bar{y}_{t}\right)+\varphi_{t}^{\top} y+p^{\top}\left(A_{t} y+B_{t} u+\psi_{t}\right) .
$$

The first order necessary optimality conditions for problem $\left(\mathcal{C} \mathcal{P}_{0}\right)$ give the existence of $\left(y_{0}, p_{0}\right) \in W^{1,1} \times W^{1,1}$ such that

$$
\begin{aligned}
\dot{y}_{0}(t) & =A_{t} y_{0}(t)+B_{t} u_{0}(t)+\psi_{t} \quad \text { for a.a. } t \in[0, T] \\
-\dot{p}_{0}(t) & =A_{t}^{\top} p_{0}(t)+C_{t}\left(y_{0}(t)-\bar{y}(t)\right)+\varphi_{t} \quad \text { for a.a. } t \in[0, T] \\
y_{0}(0) & =x_{0}, \quad p_{0}(T)=M y_{0}(T) \\
u_{0}(t) & \in \operatorname{argmin}\left\{H_{0}\left(w, y_{0}(t), p_{0}(t), t\right): w \geq 0\right\} \quad \text { for a.a. } t \in[0, T] .(6)
\end{aligned}
$$

For $(R, z) \in \mathcal{S}_{++}^{m} \times \mathbb{R}^{m}$, let us denote by $\Pi_{0}(R, z)$ the unique solution of

$$
\operatorname{Min} \frac{1}{2}(x-z)^{\top} R(x-z), \quad \text { s.t. } \quad x \in \mathbb{R}_{+}^{m} . \quad\left(\mathcal{P}_{0}^{R, z}\right)
$$

The mapping $z \rightarrow \Pi_{0}(R, z)$ simply reduces to the projection of $z$ onto $\mathbb{R}_{+}^{m}$ associated with the norm induced by the inner product $\langle x, y\rangle_{R}:=\langle R x, y\rangle$.

For all $t$ in $[0, T]$, the Hamiltonian can be rewritten as

$$
H_{0}(u, y, p, t)=g\left(u+R_{t}^{-1} B_{t}^{\top} p, y, t\right)+p^{\top}\left(A_{t} y+\psi_{t}\right)-\frac{1}{2}\left(p^{\top} B_{t}\right) R_{t}^{-1} B_{t}^{\top} p .
$$

Thus, by using (6), the optimal control may be expressed as

$$
u_{0}(t)=\Pi_{0}\left(R_{t},-R_{t}^{-1} B_{t}^{\top} p_{0}(t)\right) \quad \text { for a.a. } t \in[0, T] .
$$

Let us introduce interior penalty approximations of $\left(\mathcal{C P} \mathcal{P}_{0}\right)$. Let $\mathcal{L}$ be the class of barrier functions on $\mathbb{R}_{+}^{m}$ of the form $L(x)=\sum_{i=1}^{m} \ell\left(x^{i}\right)$, where $\ell$ is a convex function whose domain is either $\mathbb{R}_{+}$or $\mathbb{R}_{++}$, and which satisfies: $\ell$ is $\mathcal{C}^{2}$ on the interior of its domain and

$$
\text { (i) } \lim _{x \downarrow 0} \ell^{\prime}(x)=-\infty ; \quad \text { (ii) } \lim _{x \downarrow 0} \frac{\ell^{\prime \prime}(x)}{\ell^{\prime}(x)}=-\infty .
$$

Remark. Standard examples of functions satisfying these properties are:

$$
\ell(r)=-\log r \quad ; \quad \ell(r)=r \log r \quad ; \quad \ell(r)=r^{-p} \quad p>0 \quad ; \quad \ell(r)=-r^{p}, p \in(0,1) .
$$

where $r$ is a positive real number.

Note that, for $L \in \mathcal{L}$ and $u \in L^{2}$, the integral $\int_{0}^{T} L\left(u_{t}\right) \mathrm{d} t$ belongs to $\mathbb{R} \cup$ $\{+\infty\}$, since $L$, being convex over $\mathbb{R}^{n}$ with a nonempty domain, is bounded 
from below by an affine function. For $\varepsilon>0$, the perturbed cost function $J_{\varepsilon}$ : $\mathcal{U} \rightarrow \mathbb{R} \cup\{+\infty\}$ is defined as

$$
J_{\varepsilon}(u):=J_{0}(u)+\varepsilon \int_{0}^{T} L\left(u_{t}\right) \mathrm{d} t .
$$

The penalized problem is defined by

$$
\operatorname{Min} J_{\varepsilon}(u) \text {, s.t. }(1), \quad u \in \mathcal{U}_{+} .
$$

Set $\mathcal{U}_{++}:=L^{2}\left([0, T] ; \mathbb{R}_{++}^{m}\right)$. It holds that

Proposition 1. Problem $\left(\mathcal{C P}_{\varepsilon}\right)$ has a unique solution $u_{\varepsilon} \in \mathcal{U}_{++}$. Moreover, there exists a constant $K_{0}>0$ such that for all $i \in\{1, \ldots, m\}$

$$
\ell^{\prime}\left(2 u_{\varepsilon}^{i}(t)\right) \geq-\frac{2 K_{0}}{\varepsilon} \quad \text { for a.a. } t \in[0, T] .
$$

Proof. (Existence and uniqueness). Let us prove that $J_{\varepsilon}$ has a unique minimizer $u_{\varepsilon}$ in $\mathcal{U}_{+}$and then establish that $u_{\varepsilon}$ actually belongs to $\mathcal{U}_{++}$. Clearly $J_{\varepsilon}$ is strongly convex and since $\mathcal{U}_{+}$is a closed convex set of $\mathcal{U}$ it suffices to show that $J_{\varepsilon}$ is lower semicontinuous (l.s.c). Clearly this holds iff $\widehat{L}(u):=\int_{0}^{T} L\left(u_{t}\right) \mathrm{d} t$ is so. Let $\bar{u} \in \mathcal{U}_{+}$and suppose that $\widehat{L}$ is not lower semicontinuous at $\bar{u}$. Let $u_{n}$ a sequence of functions in $\mathcal{U}_{+}$converging to $\bar{u}$ such that $\widehat{L}(\bar{u})>\lim _{n \rightarrow \infty} \widehat{L}\left(u_{n}\right)$. Extracting a subsequence if necessary, we can assume that $u_{n}$ converges almost surely to $\bar{u}$. Since $L$ is convex there exists $a \in \mathbb{R}^{m}, b \in \mathbb{R}$ such that $L\left(u_{n}\right) \geq$ $a^{\top} u_{n}+b$. Applying Fatou's Lemma to the nonnegative sequence $L\left(u_{n}\right)-a^{\top} u_{n}-b$ and using the fact that $L$ is lower semicontinuous we obtain

$$
\lim _{n \rightarrow \infty} \widehat{L}\left(u_{n}\right) \geq \int_{0}^{T} \liminf _{n \rightarrow \infty} L\left(u_{n}(t)\right) \mathrm{d} t \geq \int_{0}^{T} L\left(\bar{u}_{t}\right) \mathrm{d} t=\widehat{L}(\bar{u})
$$

which yields the desired contradiction.

(Strict positivity). We obtain the result by extending the method of [7] (applied to the logarithmic penalty) to the class $\mathcal{L}$. For notational convenience we suppose that $m=1$. By (7) (i) there exists $\zeta>0$ such that $\ell$ is decreasing on $[0, \zeta]$. Set

$$
I_{\zeta}:=\left\{t \in[0, T] ; u_{\varepsilon}(t) \leq \zeta / 2\right\}
$$

and

$$
u_{\varepsilon}^{\zeta}(t):=\left\{\begin{array}{ll}
\zeta & \text { if } t \in I_{\zeta} \\
u_{\varepsilon}(t) & \text { otherwise }
\end{array} \quad ; \quad y_{\varepsilon}^{\zeta}(t):=y_{u_{\varepsilon}^{\zeta}}(t) \quad \text { for a.a. } t \in[0, T] .\right.
$$

By standard arguments $\left\|y_{\varepsilon}^{\zeta}-y_{u_{\varepsilon}}\right\|_{\infty}=O\left(\left\|u_{\varepsilon}^{\zeta}-u_{\varepsilon}\right\|_{1}\right)$. Thus, by definition of $I_{\zeta}$

$$
J_{0}\left(u_{\varepsilon}^{\zeta}\right)-J_{0}\left(u_{\varepsilon}\right)=O\left(\left\|u_{\varepsilon}^{\zeta}-u_{\varepsilon}\right\|_{1}\right)=O(\zeta) \operatorname{meas}\left(I_{\zeta}\right) .
$$

Hence there exists of $K_{0}>0$ such that

$$
J_{\varepsilon}\left(u_{\varepsilon}^{\zeta}\right)-J_{\varepsilon}\left(u_{\varepsilon}\right) \leq K_{0} \zeta \operatorname{meas}\left(I_{\zeta}\right)+\varepsilon \int_{I_{\zeta}}\left(\ell\left(u_{\varepsilon}^{\zeta}(t)\right)-\ell\left(u_{\varepsilon}(t)\right)\right) \mathrm{d} t
$$


By the mean value theorem and the convexity of $\ell$ we find that

$$
\ell\left(u_{\varepsilon}^{\zeta}(t)\right)-\ell\left(u_{\varepsilon}(t)\right) \leq \frac{1}{2} \ell^{\prime}(\zeta) \zeta
$$

for a.a. $t \in I_{\zeta}$. This in turn implies that

$$
J_{\varepsilon}\left(u_{\varepsilon}^{\zeta}\right)-J_{\varepsilon}\left(u_{\varepsilon}\right) \leq \zeta \operatorname{meas}\left(I_{\zeta}\right)\left(K_{0}+\frac{1}{2} \varepsilon \ell^{\prime}(\zeta)\right) .
$$

Therefore, by the strict optimality of $u_{\varepsilon}$, if meas $\left(I_{\zeta}\right)>0$ we have that $K_{0}>$ $-\frac{1}{2} \varepsilon \ell^{\prime}(\zeta)$. By choosing $\zeta^{\prime}$ such that $K_{0} \leq-\frac{1}{2} \varepsilon \ell^{\prime}\left(\zeta^{\prime}\right)$ we obtain that for a.a. $t \in[0, T]$

$$
\ell^{\prime}\left(2 u_{\varepsilon}(t)\right) \geq \ell^{\prime}\left(\zeta^{\prime}\right) .
$$

The conclusion follows by letting $\ell^{\prime}\left(\zeta^{\prime}\right) \uparrow-2 K_{0} / \varepsilon$.

Remark. Estimation (8) generalize the estimate $u_{\varepsilon}(t) \geq c \varepsilon$, for some $c>0$, in the case when $\ell(r)=-\log r$, obtained in [7].

For $(u, y, p, t) \in \mathbb{R}_{+}^{m} \times \mathbb{R}^{n} \times \mathbb{R}^{n} \times[0, T]$ and $\varepsilon>0$, the Hamiltonian $H_{\varepsilon}$ for the problem $\left(\mathcal{C P}_{\varepsilon}\right)$ is defined by

$$
H_{\varepsilon}(u, y, p, t):=H_{0}(u, y, p, t)+\varepsilon L(u) .
$$

The first order necessary conditions for $(\mathcal{C P} \varepsilon)$ ensure the existence of $\left(y_{\varepsilon}, p_{\varepsilon}\right) \in$ $W^{1,1} \times W^{1,1}$ such that

$$
\begin{aligned}
\dot{y}_{\varepsilon}(t) & =A_{t} y_{\varepsilon}(t)+B_{t} u_{\varepsilon}(t)+\psi_{t} \quad \text { for a.a. } t \in[0, T] \\
-\dot{p}_{\varepsilon}(t) & =A_{t}^{\top} p_{\varepsilon}(t)+C_{t}\left(y_{\varepsilon}(t)-\bar{y}(t)\right)+\varphi_{t} \quad \text { for a.a. } t \in[0, T] \\
y_{\varepsilon}(0) & =x_{0}, \quad p_{\varepsilon}(T)=M y_{\varepsilon}(T) \\
0 & =D_{u} H_{\varepsilon}\left(u_{\varepsilon}(t), y_{\varepsilon}(t), p_{\varepsilon}(t), t\right) \quad \text { for a.a. } t \in[0, T] .
\end{aligned}
$$

Condition (14) yields that $u_{\varepsilon}$ is the unique solution in $\mathcal{U}_{++}$of

$$
R_{t} u_{\varepsilon}(t)+\varepsilon \nabla L\left(u_{\varepsilon}(t)\right)=-B_{t}^{\top} p_{\varepsilon}(t) \quad \text { for a.a. } t \in[0, T] .
$$

The estimation of the error bounds related to interior penalty methods requires a detailed analysis of the analogous problem in the finite dimensional setting.

\section{Interior penalty analysis in the finite dimen- sional setting}

Given $(R, z) \in \mathcal{S}_{++}^{m} \times \mathbb{R}^{m}$ recall that $\Pi_{0}(R, z)$ is defined as the unique minimum of $f_{0}^{R, z}(x):=\frac{1}{2}(x-z)^{\top} R(x-z)$ over $\mathbb{R}_{+}^{m}$. Standard results of convex analysis ensures that $z \rightarrow \Pi_{0}(R, z)$ is nonexpansive with respect to the norm induced by $R$. $\left(\mathcal{P}_{0}^{R, z}\right)$

Given $L \in \mathcal{L}$ and $\varepsilon>0$ let us consider the following penalized version of

$$
\operatorname{Min} f_{\varepsilon}^{R, z}(x):=\frac{1}{2}(x-z)^{\top} R(x-z)+\varepsilon L(x), \quad \text { s.t. } \quad x \in \mathbb{R}_{+}^{m} . \quad\left(\mathcal{P}_{\varepsilon}^{R, z}\right)
$$

Since $f_{\varepsilon}^{R, z}$ is strongly convex and lower semicontinuous, problem $\left(\mathcal{P}_{\varepsilon}^{R, z}\right)$ has a unique solution $\Pi_{\varepsilon}(R, z)$ in $\mathbb{R}_{+}^{m}$. By a classical argument, it is easy to see that $\Pi_{\varepsilon}(R, z)$ actually belongs to $\mathbb{R}_{++}^{m}$. 


\subsection{Convergence properties of the family $\left\{\Pi_{\varepsilon}\right\}_{\epsilon \geq 0}$}

In this section, we provide several useful results on the topological and the convergence properties of the family of approximated "projectors" $\Pi_{\varepsilon}$. Some properties of the derivatives are also provided.

Lemma 2 (boundedness of $\Pi_{\varepsilon}$ ). Let $K \subseteq \mathcal{S}_{++}^{m} \times \mathbb{R}^{m}$ be a compact set. Then for $\varepsilon_{0}>0$, there is a constant $C_{1}=C_{1}\left(K, \varepsilon_{0}\right)$ such that

$$
\left|\Pi_{\varepsilon}(R, z)\right| \leq C_{1} \quad \text { for all } \varepsilon \in\left(0, \varepsilon_{0}\right) \text { and }(R, z) \in K \text {. }
$$

Proof. Let $\varepsilon \in\left(0, \varepsilon_{0}\right)$ and $y \mapsto a^{\top} y+b$ be an affine minorant of $L$. We have $\frac{1}{2}\left(\Pi_{\varepsilon}(R, z)-z\right)^{\top} R\left(\Pi_{\varepsilon}(R, z)-z\right)+\varepsilon\left(a^{\top} \Pi_{\varepsilon}(R, z)+b\right) \leq f_{\varepsilon}^{R, z}\left(\Pi_{\varepsilon}(R, z)\right) \leq f_{\varepsilon}^{R, z}(\mathbf{1})$, where $\mathbf{1} \in \mathbb{R}^{m}$ denotes the vector of ones. Since $f_{\varepsilon}^{R, z}(\mathbf{1}) \leq \max \left\{f_{0}^{R, z}(\mathbf{1}), f_{\varepsilon_{0}}^{R, z}(\mathbf{1})\right\}$, we obtain

$\frac{\lambda_{\min }(R)}{2}\left|\Pi_{\varepsilon}(R, z)-z\right|^{2}+\varepsilon\left(a^{\top} \Pi_{\varepsilon}(R, z)+b\right) \leq \sup _{\left(R^{\prime}, z^{\prime}\right) \in K} \max \left\{f_{\varepsilon_{0}}^{R^{\prime}, z^{\prime}}(\mathbf{1}), f_{0}^{R^{\prime}, z^{\prime}}(\mathbf{1})\right\}<+\infty$

implying the conclusion.

Proposition 3 (Pointwise convergence of the projectors $\Pi_{\varepsilon}$ ). Let $(R, z) \in$ $\mathcal{S}_{++}^{m} \times \mathbb{R}^{m}$, then $\lim _{\varepsilon \downarrow 0} \Pi_{\varepsilon}(R, z)=\Pi_{0}(R, z)$.

Proof. Since $(R, z)$ is fixed, we will omit it in the notation. Let $y \mapsto a^{\top} y+b$ be an affine minorant of $L$ and $c$ be a lower bound of $y \rightarrow|y|^{2}+\left(a^{\top} y+b\right)$. Since $\frac{1}{2}\left(\Pi_{\varepsilon}-z\right)^{\top} R\left(\Pi_{\varepsilon}-z\right)+\varepsilon\left(a^{\top} \Pi_{\varepsilon}+b\right) \leq f_{\varepsilon}^{R, z}\left(\Pi_{\varepsilon}\right) \leq f_{\varepsilon}^{R, z}(v), \quad$ for all $v \in \mathbb{R}_{++}^{m}$, we have

$$
\frac{1}{2}\left(\Pi_{\varepsilon}-z\right)^{\top} R\left(\Pi_{\varepsilon}-z\right)+\varepsilon c-\varepsilon\left|\Pi_{\varepsilon}\right|^{2} \leq f_{\varepsilon}^{R, z}(v), \quad \text { for all } v \in \mathbb{R}_{++}^{m} .
$$

Lemma 2 (for the particular case $K=\{(R, z)\}$ ) implies that $\Pi_{\varepsilon}$ has a cluster point $\pi_{0}$ when $\varepsilon \downarrow 0$. Passing to the limit in (17) yields $f_{0}^{R, z}\left(\pi_{0}\right) \leq f_{0}^{R, z}(v)$ for all $v \in \mathbb{R}_{++}^{m}$ and thus for all $v \in \mathbb{R}_{+}^{m}$. Hence $\pi_{0}=\Pi_{0}(R, z)$.

In order to investigate further the converge properties of $\Pi_{\varepsilon}$, it is useful to write down the first order condition for problems $\left(\mathcal{P}_{0}^{R, z}\right)$ and $\left(\mathcal{P}_{\varepsilon}^{R, z}\right)$. Let us set $\mathfrak{I}:=\{1, \ldots, m\}$. The first order condition for $\left(\mathcal{P}_{0}^{R, z}\right)$ writes

$$
\begin{aligned}
& R\left(\Pi_{0}(R, z)-z\right)-\mu(R, z)=0 \\
& \mu(R, z) \geq 0 \quad ; \quad \Pi_{0}(R, z) \geq 0 ; \quad \mu^{i}(R, z) \Pi_{0}^{i}(R, z)=0 \quad \text { for all } i \in \mathfrak{I},
\end{aligned}
$$

where $\mu(R, z)$ is the Lagrange multiplier of the problem.

On the other hand, the first order condition for $\left(\mathcal{P}_{\varepsilon}^{R, z}\right)$ shows that $\Pi_{\varepsilon}(R, z)$ is the unique solution in $\mathbb{R}_{++}^{m}$ of

$$
R\left(\Pi_{\varepsilon}(R, z)-z\right)+\varepsilon \nabla L\left(\Pi_{\varepsilon}(R, z)\right)=0 .
$$

Proposition 3 asserts that for each $z \in \mathbb{R}^{m}$ and $R \in \mathcal{S}_{++}^{m}$ the vector $\Pi_{\varepsilon}(R, z)$ converges to $\Pi_{0}(R, z)$. Actually uniform convergence holds over each compact subset of $\mathcal{S}_{++}^{m} \times \mathbb{R}^{m}$. Let us first state a preliminary lemma. 
Lemma 4 (Equicontinuity of the family $\left\{\Pi_{\varepsilon}\right\}_{\varepsilon \geq 0}$ ). For $R \in \mathcal{S}_{++}^{m}$, denote by $\kappa(R):=\|R\| / \lambda_{\min }(R)$ its condition number. Then for all $\varepsilon \geq 0$

$$
\left|\Pi_{\varepsilon}(R, y)-\Pi_{\varepsilon}(R, x)\right| \leq \kappa(R)|y-x|, \quad \text { for all } \quad x, y \in \mathbb{R}^{m}
$$

Proof. Equation (19) yields

$$
R\left(\Pi_{\varepsilon}(R, y)-\Pi_{\varepsilon}(R, x)\right)+\varepsilon\left(\nabla L\left(\Pi_{\varepsilon}(R, x)\right)-\nabla L\left(\Pi_{\varepsilon}(R, y)\right)\right)=R(y-x) .
$$

Multiplying the above by $\Pi_{\varepsilon}(R, y)-\Pi_{\varepsilon}(R, x)$ and using the monotonicity of $\nabla L$, we obtain

$$
\left(\Pi_{\varepsilon}(R, y)-\Pi_{\varepsilon}(R, x)\right)^{\top} R\left(\Pi_{\varepsilon}(R, y)-\Pi_{\varepsilon}(R, x)\right) \leq(x-y)^{\top} R\left(\Pi_{\varepsilon}(R, y)-\Pi_{\varepsilon}(R, x)\right) .
$$

Whence

$$
\lambda_{\min }(R)\left|\Pi_{\varepsilon}(R, y)-\Pi_{\varepsilon}(R, x)\right|^{2} \leq|| R|||x-y|\left|\Pi_{\varepsilon}(R, x)-\Pi_{\varepsilon}(R, y)\right|,
$$

and the conclusion follows.

Proposition 5 (First order derivatives and uniform convergence). (i) Let $\varepsilon>0$. Then the function $\Pi_{\varepsilon}: \mathcal{S}_{++}^{m} \times \mathbb{R}^{m} \rightarrow \mathbb{R}^{m}$ is of class $\mathcal{C}^{\infty}$.

(ii) Let $K_{1} \subseteq S_{++}^{m}$ be a compact set. For every $\varepsilon>0$ the partial derivative $D_{z} \Pi_{\varepsilon}(\cdot, \cdot)$ is bounded, uniformly in $\varepsilon$, over $K_{1} \times \mathbb{R}^{m}$ and is given by

$$
D_{z} \Pi_{\varepsilon}(R, z)=\left(I+\varepsilon R^{-1} \nabla^{2} L\left(\Pi_{\varepsilon}(R, z)\right)^{-1} \quad \text { for all }(R, z) \in S_{++}^{m} \times \mathbb{R}^{m} .\right.
$$

(iii) The partial derivative $D_{R} \Pi_{\varepsilon}(\cdot, \cdot)$ is bounded over compact subsets of $\mathcal{S}_{++}^{m} \times$ $\mathbb{R}^{m}$ uniformly in $\varepsilon$ and is characterized by

$$
D_{R} \Pi_{\varepsilon}(R, z) \delta R=D_{z} \Pi_{\varepsilon}(R, z) R^{-1} \delta R\left(z-\Pi_{\varepsilon}(R, z)\right) \quad \text { for all } \delta R \in \mathcal{S}^{m} .
$$

(iv) The function $\Pi_{\varepsilon}$ converges to $\Pi_{0}$ uniformly on each compact subset of $\mathcal{S}_{++}^{m} \times \mathbb{R}^{m}$.

(v) The function $(\varepsilon, R, z) \mapsto \Pi_{\varepsilon}(R, z)$ is continuous on $\mathbb{R}_{+} \times \mathcal{S}_{++}^{m} \times \mathbb{R}^{m}$.

Proof. (i) It follows from the implicit function theorem applied to (19).

(ii) Uniform boundedness is a consequence of Lemma 4, while equation (22) is obtained by differentiating (19) with respect to $z$.

(iii) Formula (23) follows from the differentiation of (19) with respect to $R$. The first assertion is then deduced from (ii) and Lemma 2.

(iv) Items (ii) and (iii) imply that the family $\left(\Pi_{\varepsilon}\right)_{\varepsilon>0}$ is equicontinuous. The result follows then from Proposition 3.

(v) Let $(\bar{R}, \bar{z}) \in \mathcal{S}_{++}^{m} \times \mathbb{R}^{m}$. The continuity of $\Pi_{\varepsilon}(R, z)$ for $\varepsilon>0$ is a consequence of the implicit function theorem. Consider now the case $\varepsilon=0$. For $\left(R^{\prime}, z^{\prime}\right),(R, z) \in \mathcal{S}_{++}^{m} \times \mathbb{R}^{m}$ we have

$$
\left|\Pi_{\varepsilon}\left(R^{\prime}, z^{\prime}\right)-\Pi_{0}(R, z)\right| \leq\left|\Pi_{\varepsilon}\left(R^{\prime}, z^{\prime}\right)-\Pi_{0}\left(R^{\prime}, z^{\prime}\right)\right|+\left|\Pi_{0}\left(R^{\prime}, z^{\prime}\right)-\Pi_{0}(R, z)\right| .
$$

By using (iv) and the fact that $\Pi_{0}$ is continuous the result readily follows. 


\subsection{Stratification results and strict complementarity re- formulations}

Fix $R \in \mathcal{S}_{++}^{m}$ and consider the following partition of $\mathfrak{I}$

$$
\begin{array}{lll}
I^{+}(R, z) & :=\left\{i \in \mathfrak{I}: \Pi_{0}^{i}(R, z)>0\right\} \\
I^{a}(R, z) & :=\left\{i \in \mathfrak{I}: \Pi_{0}^{i}(R, z)=0, \quad \mu^{i}(R, z)>0\right\} \\
I^{0}(R, z) & :=\left\{i \in \mathfrak{I}: \Pi_{0}^{i}(R, z)=0, \quad \mu^{i}(R, z)=0\right\} .
\end{array}
$$

Let us now proceed to a parametrization of the differentiability domain of the projection mapping $\Pi_{0}(R, \cdot)$. Given a subset $\Sigma$ of $\mathfrak{I}$, let us write

$$
Q_{i}:=\left\{\begin{array}{ll}
\{0\} & \text { if } i \in \Sigma, \\
\mathbb{R} & \text { if } i \in \Sigma^{c}
\end{array} \text { and } \quad Q_{\Sigma}:=\prod_{i=1}^{m} Q_{i} .\right.
$$

Every $z \in \mathbb{R}^{m}$ can be written uniquely as $z=z_{\Sigma}+z_{\Sigma^{c}}$ where $z_{\Sigma} \in Q_{\Sigma}$ and $z_{\Sigma^{c}} \in Q_{\Sigma^{c}}$. Let us define $h_{\Sigma}: \mathbb{R}_{+}^{m} \rightarrow \mathbb{R}^{m}$ as

$$
h_{\Sigma}(z)=z_{\Sigma}-R^{-1} z_{\Sigma^{c}}
$$

and set

$$
D_{\Sigma}(R):=h_{\Sigma}\left(\mathbb{R}_{++}^{m}\right) \quad ; \quad \operatorname{sing}(R):=\left(\bigcup_{\Sigma \subseteq \mathfrak{I}} D_{\Sigma}(R)\right)^{c}
$$

Optimality system (18) implies that the restriction of the mapping $z \rightarrow \Pi_{0}(R, z)$ to $D_{\Sigma}$ is the projection on the subspace $Q_{\Sigma}$ with respect to the metric induced by $R$. Note also that

$$
z=\Pi_{0}(R, z)-R^{-1} \mu(R, z)
$$

and thus

$$
D_{\Sigma}(R)=\left\{z \in \mathbb{R}^{m}: I^{+}(R, z)=\Sigma^{c}, I^{a}(R, z)=\Sigma, I^{0}(R, z)=\emptyset\right\} .
$$

Lemma 6 (Differentiability and singular sets). (i) The mapping $h_{\Sigma}$ is bijective and linear. Thus, $D_{\Sigma}(R)$ is a nonempty open convex subset of $\mathbb{R}^{m}$ and $\Pi_{0}(R, \cdot)$ is smooth on $D_{\Sigma}(R)$.

(ii) If $\Sigma_{1} \neq \Sigma_{2}$ then $D_{\Sigma_{1}}(R) \cap D_{\Sigma_{2}}(R)=\emptyset$.

(iii) For every $\bar{z} \in \operatorname{sing}(R)$ there exist $\Sigma_{1}, \Sigma_{2}$ with $\Sigma_{1} \neq \Sigma_{2}$ and $z_{n} \in D_{\Sigma_{1}}(R)$, $z_{n}^{\prime} \in D_{\Sigma_{2}}(R)$ such that $\bar{z}=\lim _{n \uparrow \infty} z_{n}=\lim _{n \uparrow \infty} z_{n}^{\prime}$. Consequently, $\Pi_{0}(R, \cdot)$ is not differentiable over $\operatorname{sing}(R)$.

Proof. (i) If $z_{\Sigma}-R^{-1} z_{\Sigma^{c}}=0$, by taking the scalar product by $z_{\Sigma^{c}}$ we obtain $z_{\Sigma^{c}}^{\top} R^{-1} z_{\Sigma^{c}}=0$ and so $z_{\Sigma^{c}}=z_{\Sigma}=0$. The second assertion follows directly since $h_{\Sigma}^{-1}$ exists and is continuous. The smoothness of $\Pi_{0}(R, \cdot)$ is straightforward since its restriction to $D_{\Sigma}(R)$ is a linear mapping.

(ii) It follows directly from characterization $(28)$ of $D_{\Sigma}(R)$.

(iii) Let $\Sigma_{1}:=I^{a}(R, \bar{z}) \cup I^{0}(R, \bar{z})$ and $z_{n}=\Pi_{0}(R, \bar{z})-R^{-1} \mu_{n}$ where $\mu_{n}^{i}=$ $1 / n$ if $i \in I^{0}(R, \bar{z})$ and $\mu_{n}^{i}=\mu^{i}(R, \bar{z})$ otherwise. Clearly, $z_{n} \in D_{\Sigma_{1}}(R)$ and $\bar{z}=\lim _{n \uparrow \infty} z_{n}$. On the other hand, let us consider $\Sigma_{2}:=I^{a}(R, \bar{z})$ and $z_{n}^{\prime}=$ $\Pi_{n}-R^{-1} \mu(R, \bar{z})$ with $\Pi_{n}^{i}=\Pi_{0}^{i}(R, \bar{z})+1 / n$ if $i \in I^{0}(R, \bar{z})$ and $\Pi_{n}^{i}=\Pi_{0}^{i}(R, \bar{z})$ otherwise. Thus, $z_{n}^{\prime} \in D_{\Sigma_{2}}(R)$ and $\bar{z}=\lim _{n \uparrow \infty} z_{n}^{\prime}$. The conclusion follows. 
Lemma 7 (Strict complementarity reformulation). For $z$ in $\mathbb{R}^{m}$, consider the linear-quadratic problem

$$
\min \left\{(x-z)^{\top} R(x-z)+c^{\top} x+d: x \in \mathbb{R}_{+}^{n}\right\},
$$

where $R, c, d$ belong respectively to $\mathcal{S}_{++}^{m}, \mathbb{R}^{m}$ and $\mathbb{R}$.

The optimal solution of this problem satisfies the strict complementarity conditions if and only if $-R^{-1} c \notin \operatorname{sing}(R)$.

Proof. Denote by $m^{*}$ the minimum value of the above problem and observe that

$$
(x-z)^{\top} R(x-z)+c^{\top} x+d=\left(x-z^{*}\right)^{\top} R\left(x-z^{*}\right)+m^{*},
$$

where $z^{*}=-R^{-1} c$. By the very definition of $\operatorname{sing}(R)$, strict complementarity holds if and only if $z^{*} \notin \operatorname{sing}(R)$.

The stratification of the domain of the projection induces a partition of the underlying matrix $R$ through the sets $I^{+}(R, z), I^{a}(R, z)$ and $I^{0}(R, z)$.

Definition 8. For $(R, z) \in \mathcal{S}_{++}^{m} \times \mathbb{R}^{m}$ define the matrices $R_{++}:=\left(R_{i, j}\right)$ where $(i, j) \in I^{+}(R, z) \times I^{+}(R, z)$ and $R_{+a}:=\left(R_{i, j}\right)$ where $(i, j) \in I^{+}(R, z) \times I^{a}(R, z)$. The matrices $R_{+0}, R_{a+}$, etc are defined similarly.

The vectors $z^{+}, z^{a}$ and $z^{0}$ are respectively obtained by removing all the coordinates of $z$ except for those in $I^{+}(R, z), I^{a}(R, z)$ and $I^{0}(R, z)$.

Proposition 9. Let $\bar{R} \in \mathcal{S}_{++}^{m}$ and $\bar{z} \in \operatorname{sing}(\bar{R})^{c}$. Then

(i) For every $i \in I^{+}(\bar{R}, \bar{z})$ it holds that $\varepsilon \ell^{\prime \prime}\left(\Pi_{\varepsilon}^{i}(R, z)\right)=O(\varepsilon)$ as $(R, z) \mapsto$ $(\bar{R}, \bar{z})$.

(ii) For every $j \in I^{a}(\bar{R}, \bar{z}), \quad \lim _{(\varepsilon, R, z) \mapsto(0, \bar{R}, \bar{z})} \varepsilon \ell^{\prime}\left(\Pi_{\varepsilon}^{j}(R, z)\right)=\mu^{j}(\bar{R}, \bar{z})>0$.

Proof. Both properties follow from (18), equation (19) and Proposition 5.

The following lemma will be instrumental for the proof of Theorem 13 .

Lemma 10. The mapping $(\varepsilon, R, z) \mapsto D_{(R, z)} \Pi_{\varepsilon}(R, z)$ is continuous in $(\bar{\varepsilon}, \bar{R}, \bar{z})$ for every $\bar{\varepsilon} \geq 0, \bar{R} \in \mathcal{S}_{++}^{m}$ and $\bar{z} \in \operatorname{sing}(\bar{R})^{c}$.

Proof. By Proposition 5 (iii) it suffices to check that $D_{z} \Pi_{\varepsilon}(R, z)$ is continuous at $(\bar{\varepsilon}, \bar{R}, \bar{z})$. The case $\bar{\varepsilon}>0$ is already proved in Proposition 5 (i).

Let us deal with the case $\bar{\varepsilon}$. Since $\bar{z} \in \operatorname{sing}(\bar{R})^{c}$ it follows that $I^{0}(\bar{z}, \bar{R})=\emptyset$. Denote

$$
\bar{I}^{+}:=I^{+}(\bar{R}, \bar{z}) \quad, \quad \bar{I}^{a}:=I^{a}(\bar{R}, \bar{z}) .
$$

Let $(R, z)$ be such that $I^{+}(R, z)=\bar{I}^{+}, I^{a}(R, z)=\bar{I}^{a}$ and $w \in \mathbb{R}^{m}$ with $|w|=1$. Let us write

$$
v_{\varepsilon}(R, z):=D_{z} \Pi_{\varepsilon}(R, z) w .
$$

For $\varepsilon>0$ equation (22) yields

$$
R v_{\varepsilon}(R, z)+\varepsilon \nabla^{2} L\left(\Pi_{\varepsilon}(R, z)\right) v_{\varepsilon}(R, z)=R w .
$$


Denote by $\operatorname{diag}_{a}\left[L^{\prime \prime}\left(\Pi_{\varepsilon}(R, z)\right)\right]$ the diagonal matrix with diagonal $\ell^{\prime \prime}\left(\Pi_{\varepsilon}^{a}(R, z)\right)$, where $\ell$ is applied componentwise. Proposition 9 (i) implies that

$$
\begin{aligned}
R_{++} v_{\varepsilon}^{+}(R, z)+R_{+a} v_{\varepsilon}^{a}(R, z)+O(\varepsilon) & =(R w)^{+} \\
R_{a+} v_{\varepsilon}^{+}(R, z)+R_{a a} v_{\varepsilon}^{a}(R, z)+\varepsilon \operatorname{diag}_{a}\left[L^{\prime \prime}\left(\Pi_{\varepsilon}(R, z)\right)\right] v_{\varepsilon}^{a}(R, z) & =(R w)^{a}
\end{aligned}
$$

For $\varepsilon$ sufficiently small the first equation of (30) implies

$$
v_{\varepsilon}^{+}(R, z)=R_{++}^{-1}(R w)^{+}-R_{++}^{-1} R_{+a} v_{\varepsilon}^{a}(R, z)+O(\varepsilon) .
$$

Set

$$
\widehat{R}^{a}:=R_{a a}-R_{a+} R_{++}^{-1} R_{+a} \quad ; \quad A_{\varepsilon}(R, z):=\widehat{R}^{a}+\varepsilon \operatorname{diag}_{a}\left[L^{\prime \prime}\left(\Pi_{\varepsilon}(R, z)\right)\right] .
$$

Substituting in the second equation of (30) the expression of $v_{\varepsilon}^{+}(R, z)$ in (31) yields

$$
A_{\varepsilon}(R, z) v_{\varepsilon}^{a}(R, z)=(R w)^{a}-R_{a+} R_{++}^{-1}(R w)^{+}+O(\varepsilon) .
$$

On the other hand, we have

$$
\lambda_{\min }\left(A_{\varepsilon}(R, z)\right) \geq \lambda_{\min }\left(\widehat{R}^{a}\right)+\min _{i \in \bar{I}^{a}} \varepsilon \ell^{\prime}\left(\Pi_{\varepsilon}^{i}(R, z)\right) \frac{\ell^{\prime \prime}\left(\Pi_{\varepsilon}^{i}(R, z)\right)}{\ell^{\prime}\left(\Pi_{\varepsilon}^{i}(R, z)\right)} .
$$

Hence proposition 9 (ii) implies that $\left\|A_{\varepsilon}^{-1}(R, z)\right\| \mapsto 0$ and as a consequence

$$
v_{\varepsilon}^{a}(R, z) \rightarrow 0=\left(D_{z} \Pi_{0}(\bar{R}, \bar{z}) w\right)^{a}
$$

uniformly in $w \in \mathbb{R}^{m},\|w\|=1$. Finally by (31)

$$
v_{\varepsilon}^{+}(R, z) \rightarrow w^{+}+\bar{R}_{++}^{-1} \bar{R}_{+a} w^{a}=\left(D_{z} \Pi_{0}(\bar{R}, \bar{z}) w\right)^{+}
$$

uniformly in $w$.

\section{Main results}

The notation are those of the previous section. For each $s \in[1,+\infty[$, we assume that the functions $\psi$ and $\varphi$ appearing respectively in the dynamics and the cost of the optimal control problem $\left(\mathcal{C P}_{0}\right)$ belong both to $L^{s}$.

Take $\varepsilon \geq 0$. In view of the optimality conditions of the problem $\left(\mathcal{C P} \mathcal{P}_{\varepsilon}\right)$, its solution $u_{\varepsilon}$ can be written as $u_{\varepsilon}(t)=\Pi_{\varepsilon}\left(R_{t},-R_{t}^{-1} B_{t}^{\top} p_{\varepsilon}(t)\right)$ where $t \in[0, T]$. Note that the curve $\left(y_{\varepsilon}, p_{\varepsilon}\right)$ belong to $W^{1, s} \times W^{1, s}$ and that the optimal control $u_{\varepsilon}$ are continuous. Consequently the optimal controls $u_{\varepsilon}$ satisfy

$$
u_{\varepsilon}(t)=\operatorname{argmin}\left\{H_{\varepsilon}\left(w, y_{\varepsilon}(t), p_{\varepsilon}(t), t\right): w \geq 0\right\}
$$

for all $t$ in $[0, T]$.

Consider the mapping

$$
F: W^{1, s} \times W^{1, s} \times \mathbb{R}_{+} \rightarrow L^{s} \times \mathbb{R}^{n} \times L^{s} \times \mathbb{R}^{n}
$$


defined by

$$
F(y, p, \varepsilon)(t):=\left(\begin{array}{c}
\dot{y}_{t}-A_{t} y_{t}-B_{t} \Pi_{\varepsilon}\left(R_{t},-R_{t}^{-1} B_{t}^{\top} p_{t}\right)-\psi_{t} \\
y_{0}-x_{0} \\
\dot{p}_{t}+A_{t}^{\top} p_{t}+C_{t}\left(y_{t}-\bar{y}_{t}\right)+\varphi_{t} \\
p_{T}-M y_{T}
\end{array}\right) .
$$

The optimality system of problem $\left(\mathcal{C P} \mathcal{P}_{\varepsilon}\right)$ may be therefore expressed, for any $\varepsilon \geq 0$, as

$$
F\left(y_{\varepsilon}, p_{\varepsilon}, \varepsilon\right)=0 \quad \text { for every } \varepsilon \geq 0 .
$$

Remark. In general, $F$ is not differentiable at $\left(y_{0}, p_{0}, 0\right)$. Indeed, take $m=$ $n=1, R_{t} \equiv 1, B_{t} \equiv 1, L(x)=-\log x$. In this case, for $p_{0} \in W^{1, s}$ and $\varepsilon \geq 0$, it holds that $\Pi_{\varepsilon}\left(1, p_{0}\right)=\phi_{\varepsilon}\left(-p_{0}\right)$ where

$$
\phi_{\varepsilon}(x):=\frac{1}{2}\left(x+\sqrt{x^{2}+4 \varepsilon}\right) .
$$

For every $t \in[0, T]$ it holds that

$$
\lim _{\varepsilon \downarrow 0} \frac{\Pi_{\varepsilon}\left(1, p_{0}(t)\right)-\Pi_{0}\left(1, p_{0}(t)\right)}{\varepsilon}= \begin{cases}\frac{1}{\left|p_{0}(t)\right|} & \text { if } p_{0}(t) \neq 0 \\ +\infty & \text { if } p_{0}(t)=0\end{cases}
$$

and generally, this limit does not belong to $L^{s}$.

In view of the above remark, a direct application of the implicit function theorem is not possible. In order to avoid this difficulty, we will use the so-called restoration theorem (see the Appendix). For $\varepsilon \geq 0$ define $\Phi_{\varepsilon}: W^{1, s} \rightarrow L^{s}$ by

$$
\Phi_{\varepsilon}(w)(t):=\Pi_{\varepsilon}\left(R_{t}, w(t)\right) .
$$

In view of Proposition 5 this function is well defined. Denote by $\# E$ the cardinality of a finite set $E$. The following two lemmas show that, under very general conditions, the assumptions of the restoration theorem are fulfilled.

Lemma 11. Let $s \in\left[1,+\infty\left[\right.\right.$ and $\widehat{w} \in W^{1, s}$ be such that

$$
\#\left\{t \in[0, T]: \widehat{w}(t) \in \operatorname{sing}\left(R_{t}\right)\right\}<+\infty .
$$

where we recall that for $R \in \mathcal{S}_{++}^{m}$ the set $\operatorname{sing}(R)$ was defined in (27). Then

(i) For every $\varepsilon>0, w \in W^{1, s}$, the function $\Phi_{\varepsilon}$ is differentiable at $w$ and for every $h \in W^{1, s}$

$$
\left(D \Phi_{\varepsilon}(w) h\right)(t)=D_{z} \Pi_{\varepsilon}\left(R_{t}, w(t)\right) h(t), \quad \text { for a.a. } t \in(0, T) .
$$

(ii) The function $\Phi_{0}$ is differentiable at $\widehat{w} \in W^{1, s}$ and for every $h \in W^{1, s}$

$$
\left(D \Phi_{\varepsilon}(\widehat{w}) h\right)(t)=D_{z} \Pi_{\varepsilon}\left(R_{t}, \widehat{w}(t)\right) h(t), \quad \text { for a.a. } t \in(0, T) .
$$

(iii) There exist a nondecreasing function $c: \mathbb{R}_{+} \rightarrow \mathbb{R}_{+}$with $\lim _{\beta \downarrow 0} c(\beta)=0$ such that for any $w^{\prime}, w \in W^{1, s}$ with $\left\|w^{\prime}-\widehat{w}\right\|_{1, s} \leq \beta, \quad\|w-\widehat{w}\|_{1, s} \leq \beta$ and $\varepsilon \in[0, \beta]$ we have

$$
\left\|\Phi_{\varepsilon}\left(w^{\prime}\right)-\Phi_{\varepsilon}(w)-D \Phi_{0}(\widehat{w})\left(w^{\prime}-w\right)\right\|\left\|_{s} \leq c(\beta)\right\| w^{\prime}-w \|_{1, s}
$$


Proof. (i) Follows directly from the implicit function theorem.

(ii) For the sake of simplicity, let us assume that $\left\{t \in[0, T]: \widehat{w}(t) \in \operatorname{sing}\left(R_{t}\right)\right\}$ reduces to the singleton $\left\{t_{0}\right\}$ and that $t_{0} \in(0, T)$; the general case follows similarly. Let $\delta>0$ be such that $\left[t_{0}-\delta, t_{0}+\delta\right] \subseteq[0, T]$. For $h \in W^{1, s}$, denote

$$
\vartheta(h)_{t}:=\left|\Pi_{0}\left(R_{t}, \widehat{w}(t)+h(t)\right)-\Pi_{0}\left(R_{t}, \widehat{w}(t)\right)-D_{z} \Pi_{0}\left(R_{t}, \widehat{w}(t)\right) h(t)\right|^{s} .
$$

We have

$$
\frac{1}{\|h\|_{1, s}^{s}}\left\|\Phi_{0}(\widehat{w}+h)-\Phi_{0}(\widehat{w})-D_{z} \Pi_{0}\left(R_{t}, \widehat{w}\right) h\right\|_{s}^{s}=I_{1}+I_{2}+I_{3},
$$

where

$$
I_{1}=\frac{1}{\|h\|_{1, s}^{s}} \int_{0}^{t_{0}-\delta} \vartheta(h)_{t} \mathrm{~d} t, \quad I_{2}=\frac{1}{\|h\|_{1, s}^{s}} \int_{t_{0}-\delta}^{t_{0}+\delta} \vartheta(h)_{t} \mathrm{~d} t, \quad I_{3}=\frac{1}{\|h\|_{1, s}^{s}} \int_{t_{0}+\delta}^{T} \vartheta(h)_{t} \mathrm{~d} t .
$$

Since $|h(t)| \leq\|h\|_{\infty} \leq c_{s}|| h \|_{1, s}$ for some $c_{s}>0$, a.a. $t \in[0, T]$, it follows that

$$
I_{1} \leq c_{s} \int_{0}^{t_{0}-\delta} \frac{\vartheta(h)_{t}}{|h(t)|^{s}} \mathrm{~d} t, \quad I_{3} \leq c_{s} \int_{t_{0}+\delta}^{T} \frac{\vartheta(h)_{t}}{|h(t)|^{s}} \mathrm{~d} t .
$$

By using Lemma 4 with $\varepsilon=0$, it follows that $\vartheta(h)_{t} /|h(t)|^{s}$ is uniformly bounded for $\|h\|_{1, s} \leq 1$ and $t \in[0, T]$. By Lebesgue's dominated convergence theorem, $I_{1}$ and $I_{3}$ vanish as $\|h\|_{1, s} \rightarrow 0$. On the other hand, using that $\Pi_{0}\left(R_{t}, z\right)$ is Lipschitz continuous uniformly in $t$ we get that $I_{2}=O(\delta)$ where the estimation does not depend on $h$ with $\|h\|_{1, s} \leq 1$. In order to obtain the claimed result, it suffices to let first $\|h\|_{1, s}$ goes to 0 and then let $\delta$ tends to 0 .

(iii) Let us first observe that

$$
\begin{gathered}
\left\|\Phi_{\varepsilon}\left(w^{\prime}\right)-\Phi_{\varepsilon}(w)-D \Phi_{0}(\widehat{w})\left(w^{\prime}-w\right)\right\|_{s}= \\
\left\|\left(\int_{0}^{1} D \Phi_{\varepsilon}\left(w+s\left(w^{\prime}-w\right)\right)-D \Phi_{0}(\widehat{w}) \mathrm{d} s\right)\left(w^{\prime}-w\right)\right\|_{s} \\
\leq \sup _{z \in B_{1, s}(\widehat{w}, \beta)}\left\|D \Phi_{\varepsilon}(z)-D \Phi_{0}(\widehat{w})\right\|_{W^{1, s} \rightarrow L^{s}}\left\|w^{\prime}-w\right\|_{1, s} .
\end{gathered}
$$

where $B_{1, s}(\widehat{w}, \beta)$ denotes the ball in $W^{1, s}$ of center $\widehat{w}$ and radius $\beta$ and $\|$. $\| W_{W^{1, s} \rightarrow L^{s}}$ denotes the standard norm of the space of linear bounded functions from $W^{1, s}$ to $L^{s}$. Let $h \in W^{1, s}$ with $\|h\|_{1, s} \leq 1$. We have

$$
\left\|D \Phi_{\varepsilon}(z) h-D \Phi_{0}(\widehat{w}) h\right\|_{s}^{s} \leq\|h\|_{\infty} \int_{0}^{T}\left|D \Pi_{\varepsilon}\left(R_{t}, z(t)\right)-D \Pi_{0}\left(R_{t}, \widehat{w}(t)\right)\right|^{s} \mathrm{~d} t
$$

and thus

$$
\sup _{z \in B_{1, s}(\widehat{w}, \beta)}\left\|D \Phi_{\varepsilon}(z)-D \Phi_{0}(\widehat{w})\right\|_{W^{1, s} \rightarrow L^{s}} \leq c(\beta)
$$

where $c(\beta)$ is defined by

$$
c(\beta):=\left(\int_{0}^{T} \sup _{\varepsilon \in[0, \beta]} \sup _{z \in B(\widehat{w}(t), \beta)}\left|D \Pi_{\varepsilon}\left(R_{t}, z\right)-D \Pi_{0}\left(R_{t}, \widehat{w}(t)\right)\right|^{s} \mathrm{~d} t\right)^{\frac{1}{s}} .
$$

In view of Proposition 5 (ii), Lemma 10, assumption (36) and Lebesgue's dominated convergence theorem, we conclude that $c(\beta) \downarrow 0$ as $\beta \downarrow 0$. 
Let us now introduce our main assumption. Recall that the control $u_{0}$ is the solution of $\left(\mathcal{C} P_{0}\right)$.

Strict complementarity assumption: There exists a finite subset $T_{\text {sing }}$ of $[0, T]$, such that for each $t$ in $[0, T] \backslash T_{\text {sing }}$ the point $u_{0}(t)$ satisfies the strict complementarity conditions for the minimization problem

$$
\min \left\{H\left(w, y_{0}(t), p_{0}(t), t\right): w \in \mathbb{R}_{+}^{n}\right\}
$$

This assumption can be reformulated in an alternative form. Note first that for almost all $t$, the control $u_{0}(t)$ actually solves the following (simplified) quadratic problem:

$$
\min \left\{v^{\top} R_{t} v+p_{0}(t)^{\top} B_{t} v: v \in \mathbb{R}_{++}^{n}\right\} .
$$

As in Lemma 7, define

$$
q_{0}(t):=-R_{t}^{-1} B_{t}^{\top} p_{0}(t)
$$

where $p_{0}$ is the adjoint state for problem $\left(\mathcal{C P}_{0}\right)$. In view of Lemma 7 , the strict complementarity assumption above exactly amounts to

$$
\#\left\{t \in[0, T]: q_{0}(t) \in \operatorname{sing}\left(R_{t}\right)\right\}=\# T_{\text {sing }}<+\infty .
$$

The following result establishes the surjectivity of the derivative of $F$ at $\left(y_{0}, p_{0}, 0\right)$ (where $F$ is defined in $\left.(32)\right)$ : this fact is central for the application of the restoration theorem (see Theorem 20).

Let us define

$$
\Sigma(t):=\mathfrak{I} \backslash I^{+}\left(R_{t}, q_{0}(t)\right), \quad \text { for all } t \in[0, T]
$$

and recall that for all $\Sigma \subseteq \mathfrak{I}$ the linear subspace $Q_{\Sigma}$ was defined in (25).

Lemma 12 (Surjectivity of the optimality mapping). Consider problems $\left(\mathcal{C P}_{0}\right)$ and $\left(\mathcal{C P}_{\varepsilon}\right)$ of Section 2. If the strict complementarity assumption (39) holds, then the function $F$ is differentiable with respect to $(y, p)$ at $\left(y_{0}, p_{0}, 0\right)$ and the linear application $D_{(y, p)} F\left(y_{0}, p_{0}, 0\right)$ is an isomorphism.

In addition, for every $\left(\delta_{1}, \delta_{2}, \delta_{3}, \delta_{4}\right) \in L^{s} \times \mathbb{R}^{n} \times L^{s} \times \mathbb{R}^{m}$, the curve

$$
D_{(y, p)} F\left(y_{0}, p_{0}, 0\right)^{-1}\left(\delta_{1}, \delta_{2}, \delta_{3}, \delta_{4}\right)
$$

is the unique solution of the reduced optimality system of

$$
\left\{\begin{array}{c}
\operatorname{Min} \frac{1}{2} \int_{0}^{T}\left(v_{t}^{\top} R_{t} v_{t}+\sigma_{t}^{\top} C_{t} \sigma_{t}-\delta_{3} \cdot \sigma_{t}\right) \mathrm{d} t \\
\quad+\frac{1}{2}\left(\sigma_{T}+M^{-1} \delta_{4}\right)^{\top} M\left(\sigma_{T}+M^{-1} \delta_{4}\right), \quad\left(\mathcal{P}_{\delta_{1}, \delta_{2}, \delta_{3}, \delta_{4}}\right) \\
\text { s.t. } \quad \dot{\sigma}_{t}=A_{t} \sigma_{t}+B_{t} v_{t}+\delta_{1}(t) \\
\sigma_{0}=\delta_{2}, \quad v_{t} \in Q_{\Sigma(t)} .
\end{array}\right.
$$

Proof. The differentiability property of $F$ is a direct consequence of Lemma 11 (ii). Now, for $\sigma$ and $\varsigma$ in $W^{1, s}$ we have

$$
D F_{(y, p)}\left(y_{0}, p_{0}, 0\right)(\sigma, \varsigma)(t)=\left(\begin{array}{c}
\dot{\sigma}_{t}-A_{t} \sigma_{t}+B_{t} D_{z} \Pi_{0}\left(R_{t}, q_{0}(t)\right) R_{t}^{-1} B_{t}^{\top} \varsigma_{t} \\
\sigma_{0} \\
\dot{\varsigma}_{t}+A_{t}^{\top} \varsigma_{t}+C_{t} \sigma_{t} \\
\varsigma_{T}-M \sigma_{T}
\end{array}\right)
$$


Let $\delta_{1} \in L^{s}, \delta_{2} \in \mathbb{R}^{n}, \delta_{3} \in L^{s}, \delta_{4} \in \mathbb{R}^{n}$ and consider the system of equations

$$
\begin{aligned}
\dot{\sigma}_{t}-A_{t} \sigma_{t}+B_{t} D_{z} \Pi_{0}\left(R_{t}, q_{0}(t)\right) R_{t}^{-1} B_{t}^{\top} \varsigma_{t} & =\delta_{1}(t) \\
\dot{\varsigma}_{t}+A_{t}^{\top} \varsigma_{t}+C_{t} \sigma_{t} & =\delta_{3}(t) \\
\varsigma_{T}-M \sigma_{T}=\delta_{4} & ; \quad \sigma_{0}=\delta_{2} .
\end{aligned}
$$

Equations (41) are the reduced first order optimality conditions of $\left(\mathcal{P}_{\delta_{1}, \delta_{2}, \delta_{3}, \delta_{4}}\right)$. This last problem is strongly convex and consequently it has a unique solution $(\bar{\sigma}, \bar{\varsigma}) \in W^{1, s} \times W^{1, s}$.

$\mathbf{W}^{1, \infty}$ assumption: We shall say that $W^{1, \infty}$ assumption holds if:

- $A \in W^{1, \infty}\left([0, T] ; \mathbb{R}^{n \times n}\right)$,

- $B \in W^{1, \infty}\left([0, T] ; \mathbb{R}^{n \times m}\right)$,

- $R \in W^{1, \infty}\left([0, T] ; \mathcal{S}_{++}^{m}\right)$,

- $C \in W^{1, \infty}\left([0, T] ; \mathcal{S}_{+}^{n}\right)$.

Clearly, under this assumption, $u_{\varepsilon} \in W^{1, \infty}$ for all $\varepsilon \geq 0$.

Recall that the function $\Phi_{\varepsilon}: W^{1, s} \rightarrow L^{s}$ is defined as

$$
\Phi_{\varepsilon}(w)(t):=\Pi_{\varepsilon}\left(R_{t}, w(t)\right), \quad t \in[0, T] .
$$

For each fixed $t$, the quantity $\left|\Phi_{\varepsilon}(w)(t)-\Phi_{0}(w)(t)\right|$ therefore measures the error estimate of the penalty method for the finite dimensional problem

$$
\min \left\{(x-w(t))^{\top} R_{t}(x-w(t)): x \in \mathbb{R}_{+}^{m}\right\} .
$$

The following result shows that these finite dimensional error bounds can be used to recover the error bounds for the penalized optimal control problem $(\mathcal{C P})_{\varepsilon}$.

Theorem 13 (Error estimates for interior penalty). Let $s$ be in $[1,+\infty)$, suppose that $\psi$ and $\varphi$ belong to $L^{s}$. and assume further that the strict complementarity assumption (39) and the $W^{1, \infty}$ assumption hold. Then

(i) The error estimates for $u_{\varepsilon}, y_{\varepsilon}$ and $p_{\varepsilon}$ are given by

$$
\left\|u_{\varepsilon}-u_{0}\right\|_{s}+\left\|y_{\varepsilon}-y_{0}\right\|_{1, s}+\left\|p_{\varepsilon}-p_{0}\right\|_{1, s}=O\left(\left\|\Phi_{\varepsilon}\left(q_{0}\right)-\Phi_{0}\left(q_{0}\right)\right\|_{s}\right),
$$

with in addition $u_{\varepsilon} \rightarrow u_{0}$ in $W^{1, s}$.

(ii) The error bound for the control in the infinity norm is given by

$$
\left\|u_{\varepsilon}-u_{0}\right\|_{\infty}=O\left(\left\|\Phi_{\varepsilon}\left(q_{0}\right)-\Phi_{0}\left(q_{0}\right)\right\|_{\infty}\right) .
$$

(iii) The error estimates for the cost is given by

$$
\left|J_{0}\left(u_{\varepsilon}\right)-J_{0}\left(u_{0}\right)\right|=O\left(\left\|\Phi_{\varepsilon}\left(q_{0}\right)-\Phi_{0}\left(q_{0}\right)\right\|_{1}\right) .
$$


Proof. First, for $\varepsilon>0$ let us define

$$
q_{\varepsilon}(t):=-R_{t}^{-1} B_{t}^{\top} p_{\varepsilon}(t) \text { for all } t \in[0, T]
$$

(i) In view of Lemma 11 and Lemma 12 the mapping $F$ defined in (32)(page 13), satisfies the assumptions of the restoration theorem. Therefore

$$
\left\|y_{\varepsilon}-y_{0}\right\|_{1, s}+\left\|p_{\varepsilon}-p_{0}\right\|_{1, s}=O\left(\left\|F\left(y_{0}, p_{0}, \varepsilon\right)\right\|_{s}\right)
$$

But

$$
\left\|F\left(y_{0}, p_{0}, \varepsilon\right)\right\|_{s}=\left\|F\left(y_{0}, p_{0}, \varepsilon\right)-F\left(y_{0}, p_{0}, 0\right)\right\|_{s}=O\left(\left\|\Phi_{\varepsilon}\left(q_{0}\right)-\Phi_{0}\left(q_{0}\right)\right\|_{s}\right)
$$

On the other hand, for every $t \in[0, T]$

$$
\begin{gathered}
\left|u_{\varepsilon}(t)-u_{0}(t)\right|=\left|\Pi_{\varepsilon}\left(R_{t}, q_{\varepsilon}(t)\right)-\Pi_{0}\left(R_{t}, q_{0}(t)\right)\right| \\
\leq\left|\Pi_{\varepsilon}\left(R_{t}, q_{\varepsilon}(t)\right)-\Pi_{\varepsilon}\left(R_{t}, q_{0}(t)\right)\right|+\left|\Pi_{\varepsilon}\left(R_{t}, q_{0}(t)\right)-\Pi_{0}\left(R_{t}, q_{0}(t)\right)\right|
\end{gathered}
$$

Therefore, Lemma 4 implies that

$$
\left|u_{\varepsilon}(t)-u_{0}(t)\right| \leq \kappa\left(R_{t}\right)\left|q_{\varepsilon}(t)-q_{0}(t)\right|+\left|\Pi_{\varepsilon}\left(R_{t}, q_{0}(t)\right)-\Pi_{0}\left(R_{t}, q_{0}(t)\right)\right|
$$

and the first assertion follows by taking the $L^{s}$ norm.

Let us prove the second assertion. Since the convergence of $u_{\varepsilon}$ to $u_{0}$ in $L^{s}$ is already established, it suffices to prove the convergence in $L^{s}$ of the derivatives. For almost all $t \in[0, T]$, we have that

$$
\left|\dot{u}_{\varepsilon}(t)-\dot{u}_{0}(t)\right|=\Delta_{1}(t)+\Delta_{2}(t)
$$

where

$$
\Delta_{1}(t):=\left(D_{R} \Pi_{\varepsilon}\left(R_{t}, q_{\varepsilon}(t)\right)-D_{R} \Pi_{0}\left(R_{t}, q_{0}(t)\right)\right) \dot{R}_{t}
$$

and

$$
\Delta_{2}(t):=D_{z} \Pi_{\varepsilon}\left(R_{t}, q_{\varepsilon}(t)\right) \dot{q}_{\varepsilon}(t)-D_{z} \Pi_{0}\left(R_{t}, q_{0}(t)\right) \dot{q}_{0}(t) .
$$

The convergence of $\Delta_{1}$ to 0 in $L^{s}$ follows from Lemma 10 and Lebesgue dominated convergence theorem. As for $\Delta_{2}$, let us first rewrite $\Delta_{2}(t)$ as

$$
D_{z} \Pi_{\varepsilon}\left(R_{t}, q_{\varepsilon}(t)\right)\left(\dot{q}_{\varepsilon}(t)-\dot{q}_{0}(t)\right)+D_{z} \Pi_{\varepsilon}\left(R_{t}, q_{\varepsilon}(t)\right)\left(\dot{q}_{0}(t)\right)-D_{z} \Pi_{0}\left(R_{t}, q_{0}(t)\right) \dot{q}_{0}(t) .
$$

and apply once more Lemma 10 and Lebesgue theorem.

(ii) Equation (46) implies that

$$
\left\|u_{\varepsilon}-u_{0}\right\|_{\infty} \leq \sup _{t \in[0, T]} \kappa\left(R_{t}\right)\left\|q_{\varepsilon}-q_{0}\right\|_{\infty}+\left\|\Phi_{\varepsilon}\left(q_{0}\right)-\Phi_{0}\left(q_{0}\right)\right\|_{\infty}
$$

Since

$\left\|q_{\varepsilon}-q_{0}\right\|_{\infty}=O\left(\left\|p_{\varepsilon}-p_{0}\right\|_{1, s}\right)=O\left(\left\|\Phi_{\varepsilon}\left(q_{0}\right)-\Phi_{0}\left(q_{0}\right)\right\|_{s}\right)=O\left(\left\|\Phi_{\varepsilon}\left(q_{0}\right)-\Phi_{0}\left(q_{0}\right)\right\|_{\infty}\right)$,

the result follows from (i).

(iii) By using (i), we obtain

$$
\left|J_{0}\left(u_{\varepsilon}\right)-J_{0}\left(u_{0}\right)\right|=O\left(\left\|u_{\varepsilon}-u_{0}\right\|_{1}\right)=O\left(\left\|\Phi_{\varepsilon}\left(q_{0}\right)-\Phi_{0}\left(q_{0}\right)\right\|_{1}\right) .
$$


The use of the restoration theorem also provides asymptotic expansions for the state and the adjoint state.

Theorem 14 (Asymptotic expansions). Assume that $\psi$ and $\varphi$ belong to $L^{s}$ where $s \in[1,+\infty)$. Suppose that the strict complementarity assumption (39) holds. Then

$\left(\begin{array}{c}y_{\varepsilon} \\ p_{\varepsilon}\end{array}\right)=\left(\begin{array}{c}y_{0} \\ p_{0}\end{array}\right)+D_{(y, p)} F\left(y_{0}, p_{0}, 0\right)^{-1} F\left(y_{0}, p_{0}, \varepsilon\right)+r(\varepsilon) ; \quad r(\varepsilon)=o\left(\left\|F\left(y_{0}, p_{0}, \varepsilon\right)\right\|_{s}\right)$.

Moreover, $D_{(y, p)} F\left(y_{0}, p_{0}, 0\right)^{-1} F\left(y_{0}, p_{0}, \varepsilon\right)$ is given by the solution of

$$
\left\{\begin{array}{l}
\operatorname{Min} \frac{1}{2} \int_{0}^{T}\left(v_{t}^{\top} R_{t} v_{t}+\sigma_{t}^{\top} C_{t} \sigma_{t}\right) \mathrm{d} t+\frac{1}{2} \sigma_{T}^{\top} M \sigma_{T}, \\
\text { s.t. } \\
\dot{\sigma}_{t}=A_{t} \sigma_{t}+B_{t} v_{t}-B_{t}\left(\Pi_{\varepsilon}\left(R_{t}, q_{0}(t)\right)-\Pi_{0}\left(R_{t}, q_{0}(t)\right)\right) \\
\sigma_{0}=0, \quad v_{t} \in Q_{\Sigma(t)} .
\end{array}\right.
$$

Proof. Since for every $t \in[0, T]$

$F\left(y_{0}, p_{0}, \varepsilon\right)_{t}=F\left(y_{0}, p_{0}, \varepsilon\right)_{t}-F\left(y_{0}, p_{0}, 0\right)_{t}=\left(\begin{array}{c}-B_{t}\left(\Pi_{\varepsilon}\left(R_{t}, q_{0}(t)\right)-\Pi_{0}\left(R_{t}, q_{0}(t)\right)\right) \\ 0 \\ 0 \\ 0\end{array}\right)$

the result follows directly from Corollary 21 taking $\varepsilon=\beta$ and lemma 12 taking $\delta_{1}=-B_{t}\left(\Pi_{\varepsilon}\left(R_{t}, q_{0}(t)\right)-\Pi_{0}\left(R_{t}, q_{0}(t)\right)\right), \delta_{2}=0, \delta_{3}=0$ and $\delta_{4}=0$.

\section{$5 \quad$ Examples}

As it appears from the following examples, Theorem 13 can be used to reduce the estimation of error bounds of an Optimal control problem to standard computations used in Mathematical Programming.

\subsection{Decoupled case: $R_{t} \equiv I$}

Since $R$ is no longer a variable, we simply write $\Pi_{\varepsilon}(z)$ for $\Pi_{\varepsilon}(R, z)$. In this case one has

$$
D \Pi_{\varepsilon}(z)=\left(I+\varepsilon \nabla^{2} L\left(\Pi_{\varepsilon}(z)\right)\right)^{-1} \succ 0 .
$$

Set

$I^{+}(z)=\left\{i \in \mathfrak{I}: z_{i}>0\right\} ; I^{a}(z)=\left\{i \in \mathfrak{I}: z_{i}<0\right\} ; I^{0}(z)=\left\{i \in \mathfrak{I}: z_{i}=0\right\}$.

Clearly $D \Pi_{\varepsilon}(z)$ is a positive-definite diagonal matrix. Therefore, for every $i \in \mathfrak{I}$ the function $\left(\Pi_{\varepsilon}\right)_{i}$ is non-decreasing with $z_{i}$ and constant with respect to $z_{j}$ for $j \neq i$. This implies that

$$
\left\|\Pi_{\varepsilon}^{i}(z)-\Pi_{0}^{i}(z)\right\|=\left\|\Pi_{\varepsilon}^{i}(z)\right\| \leq\left\|\Pi_{\varepsilon}^{i}(0)\right\| \quad \text { for all } z \in \mathbb{R}^{m}, i \in I^{a}(z) \cup I^{0}(z) .
$$

On the other hand, equations (19) and (18) give

$$
\Pi_{\varepsilon}^{+}(z)+\varepsilon \nabla L\left(\Pi_{\varepsilon}^{+}(z)\right)=z^{+} \quad ; \quad \Pi_{0}^{+}(z)=z^{+}
$$


and so $D\left(\Pi_{\varepsilon}^{+}-\Pi_{0}^{+}\right)(z)=-\varepsilon \nabla^{2} L\left(\Pi_{\varepsilon}^{+}(z)\right) D \Pi_{\varepsilon}^{+}(z) \leq 0$. Therefore

$$
\left\|\Pi_{\varepsilon}^{+}(z)-\Pi_{0}^{+}(z)\right\| \leq\left\|\Pi_{\varepsilon}^{+}(0)-\Pi_{0}^{+}(0)\right\|=\left\|\Pi_{\varepsilon}^{+}(0)\right\| .
$$

Finally, Theorem 13 (iv) together with equations (49) and (50) imply that

$$
\left\|u_{\varepsilon}-u_{0}\right\|_{\infty}=O\left(\left\|\Pi_{\varepsilon}(0)\right\|\right) .
$$

Let us now compute $\left\|\Pi_{\varepsilon}(0)\right\|$ for some specific barriers.

\subsubsection{Negative power penalty}

For the negative power penalty $\ell(x)=x^{-p}$, (with $p>0$ ), we obtain $\Pi_{\varepsilon}(0)-$ $p \varepsilon / \Pi_{\varepsilon}(0)^{p+1}=0$ by taking $z=0$ in (19). Therefore $\Pi_{\varepsilon}(0)=p^{\frac{1}{2+p}} \varepsilon^{\frac{1}{2+p}} \mathbf{1}$. Conclude with (51) that

$$
\left\|u_{\varepsilon}-u_{0}\right\|_{\infty}=O\left(\varepsilon^{\frac{1}{2+p}}\right) .
$$

The next example shows that the logarithmic barrier provides a smaller $L^{\infty}$ error bound, and even more importantly, a considerably better and sharper bound for the $L^{1}$ norm.

\subsubsection{Logarithmic penalty}

The logarithmic penalty corresponds to the choice $\ell(x)=-\log x$. By taking $z=0$ in (19), we get $\Pi_{\varepsilon}(0)-\varepsilon / \Pi_{\varepsilon}(0)=0$. Therefore $\Pi_{\varepsilon}(0)=\sqrt{\varepsilon} \mathbf{1}$ and, thus (51) yields

$$
\left\|u_{\varepsilon}-u_{0}\right\|_{\infty}=O(\sqrt{\varepsilon}) .
$$

In the present case, equation (19) writes

$$
\Pi_{\varepsilon}^{i}(z)=\frac{1}{2}\left(z^{i}+\sqrt{\left(z^{i}\right)^{2}+4 \varepsilon}\right)=\phi_{\varepsilon}\left(z^{i}\right) \quad \text { for all } z \in \mathbb{R}^{m}, \quad i \in \mathfrak{I} .
$$

where $\phi_{\varepsilon}$ is defined as in (33).

The family $\left(\phi_{\varepsilon}\right)_{0 \leq \varepsilon<\infty}$ enjoy several properties which can be easily established by the reader.

Lemma 15 (The $\phi_{\varepsilon}$ family). For every $\varepsilon>0$ :

(i) The function $s \mapsto \phi_{\varepsilon}(s)-\phi_{0}(s)$ is even, increasing in $(-\infty, 0)$ (and decreasing in $(0,+\infty))$.

(ii) A primitive of $\phi_{\varepsilon}$ is given by

$$
\Psi_{\varepsilon}(x)=\frac{1}{4} x^{2}+\frac{1}{4} x \sqrt{x^{2}+4 \varepsilon}+\varepsilon \log \left(x+\sqrt{x^{2}+4 \varepsilon}\right) .
$$

(iii) For every $s>0$ and $x \in \mathbb{R}, \quad \phi_{s \varepsilon}(s x)=\sqrt{s} \phi_{\varepsilon}(\sqrt{s} x)$.

The following lemma is fundamental for the error estimation in the $L^{1}$ norm.

Lemma 16. Let $q \in C([0, T])$. Assume that $Z(q):=\{t \in[0, T]: q(t)=0\}$ is finite and that for every $s_{0} \in Z(q)$ the curve $q$ is differentiable at $s_{0}$ with $\frac{\mathrm{d} q}{\mathrm{~d} t}\left(s_{0}\right) \neq 0$. Then

$$
\int_{0}^{T}\left(\phi_{\varepsilon}(q(t))-\phi_{0}(q(t))\right) \mathrm{d} t=O(\varepsilon \log \varepsilon)
$$



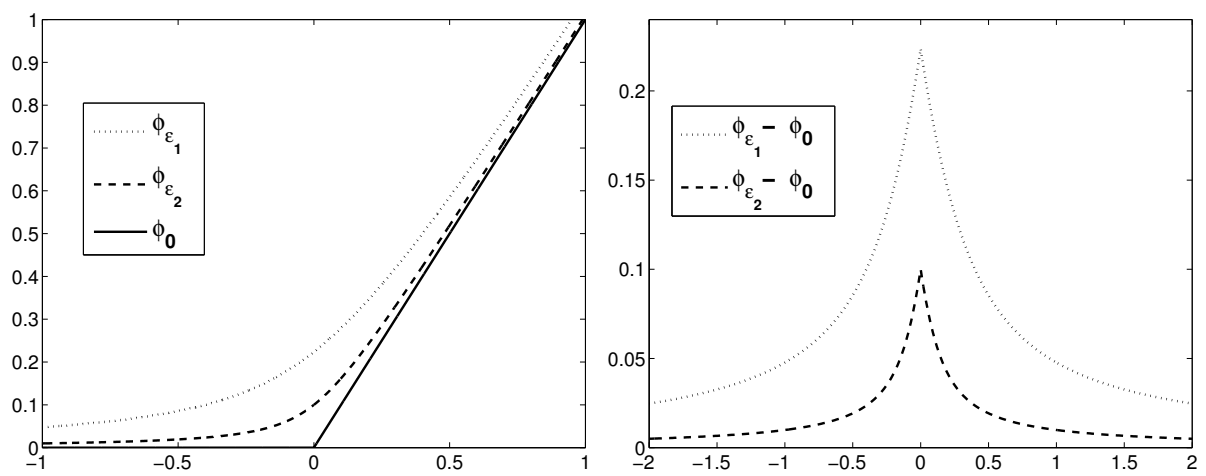

Figure 1: Left: $\phi_{\varepsilon_{1}}, \phi_{\varepsilon_{2}}$ and $\phi_{0}$. Right: $\phi_{\varepsilon_{1}}-\phi_{0}, \phi_{\varepsilon_{2}}-\phi_{0}$, for $\varepsilon_{1}=0.005$, $\varepsilon_{2}=0.001$.

Proof. With no loss of generality, let us assume that $Z(q)=\left\{s_{0}\right\}$ and that $\frac{\mathrm{d} q}{\mathrm{~d} t}\left(s_{0}\right)>0$. We have

$$
\int_{0}^{T}\left(\phi_{\varepsilon}(q(t))-\phi_{0}(q(t))\right) d s=A_{1}+B_{1}
$$

where

$A_{1}=\int_{\{t: q(t)>0\}}\left(\phi_{\varepsilon}(q(t))-\phi_{0}(q(t))\right) d s \& B_{1}=\int_{\{t: q(t)<0\}}\left(\phi_{\varepsilon}(q(t))-\phi_{0}(q(t))\right) d s$.

Since $\phi_{\varepsilon}-\phi_{0}$ is even, it suffices to obtain an estimation for $A_{1}$. Note that $\{t: q(t)>0\}=\left(s_{0}, T\right]$ since we are assuming that $Z(q)=\left\{s_{0}\right\}$. Since $\frac{d q}{\mathrm{~d} t}\left(s_{0}\right)>0$, there exists $a>0$ such that $q(s) \geq a\left(s-s_{0}\right)>0$ for all $s \in\left[s_{0}, T\right]$. On the other hand, by Lemma 15 (i) the function $s \rightarrow \phi_{\varepsilon}(s)-\phi_{0}(s)$ is decreasing in $] 0,+\infty[$ and so

$$
A_{1} \leq \int_{s_{0}}^{T}\left(\phi_{\varepsilon}\left(a\left(s-s_{0}\right)\right)-\phi_{0}\left(a\left(s-s_{0}\right)\right)\right) d s=\frac{1}{a} \int_{0}^{c}\left(\phi_{\varepsilon}(s)-s\right) d d s
$$

where $c:=a\left(T-s_{0}\right)$. By Lemma 15 (ii)

$$
\begin{aligned}
\int_{0}^{c}\left(\phi_{\varepsilon}(s)-s\right) & \\
d d s & =-\frac{c^{2}}{4}+\frac{c}{4} \sqrt{c^{2}+4 \varepsilon}+\varepsilon \log \left(c^{2}+\sqrt{c^{2}+4 \varepsilon}\right)-\varepsilon \log \sqrt{4 \varepsilon} \\
& \leq \frac{c}{4}\left(\frac{4 \varepsilon}{c+\sqrt{c^{2}+4 \varepsilon}}\right)+O(\varepsilon|\log \varepsilon|)=O(\varepsilon|\log \varepsilon|) .
\end{aligned}
$$

By combining Theorem 13 and Lemma 16, one obtains:

Theorem 17. Consider problems $\left(\mathcal{C P}_{0}\right)$ and $\left(\mathcal{C P}_{\varepsilon}\right)$, with $R_{t} \equiv I$ and $\ell(x)=$ $-\log (x)$. Suppose that the strict complementarity conditions (39) and $W^{1, \infty}$ 
assumption hold. Then:

(i) We have that

$$
\begin{gathered}
\left\|u_{\varepsilon}-u_{0}\right\|_{\infty}+\left\|p_{\varepsilon}-p_{0}\right\|_{\infty}+\left\|y_{\varepsilon}-y_{0}\right\|_{\infty}=O(\sqrt{\varepsilon}), \\
\left|J_{0}\left(u_{\varepsilon}\right)-J_{0}\left(u_{0}\right)\right|=O(\sqrt{\varepsilon}) .
\end{gathered}
$$

(ii) If in addition we assume that

$$
\left(q_{0}\left(t_{0}\right)\right)^{k}=0 \Rightarrow B \text { is differentiable at } t_{0} \text { and } \frac{\mathrm{d}}{\mathrm{d} t}\left(q_{0}\left(t_{0}\right)\right)^{k} \neq 0 .
$$

Then

$$
\begin{gathered}
\left\|u_{\varepsilon}-u_{0}\right\|_{1}+\left\|p_{\varepsilon}-p_{0}\right\|_{1,1}+\left\|y_{\varepsilon}-y_{0}\right\|_{1,1}=O(\varepsilon|\log \varepsilon|), \\
\left|J_{0}\left(u_{\varepsilon}\right)-J_{0}\left(u_{0}\right)\right|=O(\varepsilon|\log \varepsilon|) .
\end{gathered}
$$

Remark. The exact computations performed in [1] for a specific problem show that the bounds provided in (ii) are optimal.

\subsection{Coupled case: $R_{t} \succ 0$}

Recall that $u_{0}(t)=\Pi_{0}\left(R_{t}, q_{0}(t)\right)$. Roughly speaking our hypothesis is that:

- $q_{0}(t)$ meets the singular region $\operatorname{sing}\left(R_{t}\right)$ for a finite sequence of times

- when the singular region is met at most one inactive (active) constraint can become active (inactive).

This assumption allows, after a localization argument, to write $\left\|\Pi_{\varepsilon}-\Pi_{0}\right\|$ in terms of $\left|\phi_{\varepsilon}-\phi_{0}\right|$ (see Subsection 5.1).

Consider again problems $\left(\mathcal{P}_{0}^{R, z}\right)$ and $\left(\mathcal{P}_{\varepsilon}^{R, z}\right)$ as defined in Section 2 and 3 respectively. We say that $z \in \operatorname{sing}(R)$ is a simple singular point if $I^{0}(R, z)$ is a singleton. Let $K_{1} \subseteq \mathcal{S}_{++}^{m}$ and $K_{2} \subseteq \mathbb{R}^{m}$ be compacts sets. Suppose that there exists $k \in \mathfrak{I}$ such that for all $R \in K_{1}$

$$
z \in K_{2} \cap \operatorname{sing}(R) \Rightarrow z \text { is a simple singular point and } I^{0}(R, z)=\{k\}
$$

(i.e. $K_{1}$ contains only simple singular points and the active constraint with null multiplier is the same for all of them). Let $(\bar{R}, \bar{z}) \in K_{1} \times K_{2}$ such that $\bar{z} \in K_{2} \cap \operatorname{sing}(R)$ and partition $(R, z) \in K_{1} \times K_{2}$ according to $I^{+}(\bar{R}, \bar{z}), I^{a}(\bar{R}, \bar{z})$ and $I^{0}(\bar{R}, \bar{z})$. For all $(R, z) \in K_{1} \times K_{2}$, set

$$
r^{k}(R, z):=(R z)^{k}-R_{k+} R_{++}^{-1}(R z)^{+}
$$

where

$$
\widehat{R}^{k}:=R_{k k}-R_{k+} R_{++}^{-1} R_{+k}
$$

Lemma 18. It holds that

$$
\begin{aligned}
\left\|\Pi_{\varepsilon}^{+}(R, z)-\Pi_{0}^{+}(R, z)\right\| \leq & \frac{1}{\sqrt{\widehat{R}^{k}}}\left\|R_{+k}\right\|\left(\phi_{\varepsilon}\left(\frac{r^{k}(R, z)}{\sqrt{\widehat{R}^{k}}}\right)-\phi_{0}\left(\frac{r^{k}(R, z)}{\sqrt{\widehat{R}^{k}}}\right)\right)+O(\varepsilon) \\
& \left\|\Pi_{\varepsilon}^{a}(R, z)-\Pi_{0}^{a}(R, z)\right\|=O(\varepsilon) \\
\left|\Pi_{\varepsilon}^{k}(R, z)-\Pi_{0}^{k}(R, z)\right| & =\frac{1}{\sqrt{\widehat{R}^{k}}}\left(\phi_{\varepsilon}\left(\frac{r^{k}(R, z)}{\sqrt{\widehat{R}^{k}}}\right)-\phi_{0}\left(\frac{r^{k}(R, z)}{\sqrt{\widehat{R}^{k}}}\right)\right)+O(\varepsilon)
\end{aligned}
$$

where the bounds $O(\varepsilon)$ are uniform on $K_{1} \times K_{2}$. 
Proof. Let $(R, z) \in K_{1} \times K_{2}$ we have

$$
\begin{aligned}
& R_{++} \Pi_{\varepsilon}^{+}(R, z)+R_{+k} \Pi_{\varepsilon}^{k}(R, z)-\frac{\varepsilon}{\Pi_{k}^{+}(R, z)}=(R z)^{+}+O(\varepsilon) \\
& R_{k+} \Pi_{\varepsilon}^{+}(R, z)+R_{k k} \Pi_{\varepsilon}^{k}(R, z)-\frac{\varepsilon}{\Pi_{\varepsilon}^{k}(R, z)}=(R z)^{k}+O(\varepsilon),
\end{aligned}
$$

where the bounds $O(\varepsilon)$ are uniform on $K_{1} \times K_{2}$ and correspond to the terms with indices in $I^{a}(\bar{R}, \bar{z})$. From the first equation we obtain

$$
\Pi_{\varepsilon}^{+}(R, z)=R_{++}^{-1}\left((R z)^{+}-R_{+k} \Pi_{\varepsilon}^{k}(R, z)\right)+O(\varepsilon) .
$$

Substituting $\Pi_{\varepsilon}^{+}(R, z)$ in the second equation of $(65)$, we find

$$
\widehat{R}^{k} \Pi_{\varepsilon}^{k}(R, z)-\frac{\varepsilon}{\Pi_{\varepsilon}^{k}(R, z)}=r^{k}(R, z)+O(\varepsilon) .
$$

Lemma 15 (iii) yields

$$
\Pi_{\varepsilon}^{k}(R, z)=\frac{1}{\sqrt{\widehat{R}^{k}}} \phi_{\varepsilon}\left(\frac{r^{k}(R, z)}{\sqrt{\widehat{R}^{k}}}+O(\varepsilon)\right)=\frac{1}{\sqrt{\widehat{R}^{k}}} \phi_{\varepsilon}\left(\frac{r^{k}(R, z)}{\sqrt{\widehat{R}^{k}}}\right)+O(\varepsilon) .
$$

Since

$$
\Pi_{0}^{k}(R, z)=\frac{1}{\sqrt{\widehat{R}^{k}}} \phi_{0}\left(\frac{r^{k}(R, z)}{\sqrt{\widehat{R}^{k}}}\right)
$$

we have that

$$
\left|\Pi_{\varepsilon}^{k}(R, z)-\Pi_{0}^{k}(R, z)\right|=\frac{1}{\sqrt{\widehat{R}^{k}}}\left(\phi_{\varepsilon}\left(\frac{r^{k}(R, z)}{\sqrt{\widehat{R}^{k}}}\right)-\phi_{0}\left(\frac{r^{k}(R, z)}{\sqrt{\widehat{R}^{k}}}\right)\right)+O(\varepsilon)
$$

and that

$\left\|\Pi_{\varepsilon}^{+}(R, z)-\Pi_{0}^{+}(R, z)\right\| \leq \frac{1}{\sqrt{\widehat{R}^{k}}}\left\|R_{+k}\right\|\left(\phi_{\varepsilon}\left(\frac{r^{k}(R, z)}{\sqrt{\widehat{R}^{k}}}\right)-\phi_{0}\left(\frac{r^{k}(R, z)}{\sqrt{\widehat{R}^{k}}}\right)\right)+O(\varepsilon)$

which yields the result.

Theorem 19. Consider the problems $\left(\mathcal{C P}_{0}\right)$ and $\left(\mathcal{C P} \mathcal{P}_{\varepsilon}\right)$ with $\ell(x)=-\log (x)$. Suppose that the strict complementarity (39) and the $W^{1, \infty}$ assumption hold with in addition

$$
q_{0}\left(t_{0}\right) \in \operatorname{sing}\left(R_{t_{0}}\right) \Rightarrow \quad q_{0}\left(t_{0}\right) \text { is a simple singular point. }
$$

Then (i) The following holds:

$$
\begin{gathered}
\left\|u_{\varepsilon}-u_{0}\right\|_{\infty}+\left\|p_{\varepsilon}-p_{0}\right\|_{\infty}+\left\|y_{\varepsilon}-y_{0}\right\|_{\infty}=O(\sqrt{\varepsilon}) \\
\left|J_{0}\left(u_{\varepsilon}\right)-J_{0}\left(u_{0}\right)\right|=O(\sqrt{\varepsilon})
\end{gathered}
$$

(ii) If in addition we suppose that

$$
I^{0}\left(R_{t_{0}}, q_{0}\left(t_{0}\right)\right)=\{k\} \Rightarrow B \text { is differentiable at } t_{0} \text { and } \frac{\mathrm{d}}{\mathrm{d} t} r^{k}\left(R_{t_{0}}, q_{0}\left(t_{0}\right)\right) \neq 0 .
$$

Then

$$
\begin{gathered}
\left\|u_{\varepsilon}-u_{0}\right\|_{1}+|| p_{\varepsilon}-p_{0}\left\|_{1,1}+\right\| y_{\varepsilon}-y_{0} \|_{1,1}=O(\varepsilon|\log \varepsilon|), \\
\left|J_{0}\left(u_{\varepsilon}\right)-J_{0}\left(u_{0}\right)\right|=O(\varepsilon|\log \varepsilon|) .
\end{gathered}
$$


Remark. (a) If $t_{0} \in[0, T]$ is such that $I^{0}\left(R_{t_{0}}, q_{0}\left(t_{0}\right)\right)=\{k\}$ then, by taking $\varepsilon \downarrow 0$ in (66) we see that $r^{k}\left(R_{t_{0}}, q_{0}\left(t_{0}\right)\right)=0$. Thus assumption (70) is an extension for the coupled case of (59).

(b) In view of the exact computations performed in [1] for a specific problem, the bounds provided in (ii) are optimal.

Proof. Item (i) is a direct consequence of Theorem 13, while (ii) follows from Theorem 13, Lemma 18 and Lemma 16.

\section{Appendix: Proof of the Restoration Theorem}

For the sake of completeness we reproduce in this section some of the material in $[1]$.

Theorem 20. (Restoration theorem) Let $X$ and $Y$ be Banach spaces, $E$ a metric space and $F: U \subset X \times E \rightarrow Y$ a continuous mapping on an open set $U$. Let $\left(\hat{x}, \varepsilon_{0}\right) \in U$ be such that $F\left(\hat{x}, \varepsilon_{0}\right)=0$. Assume that there exists a surjective linear continuous mapping $A: X \rightarrow Y$ and a function $c: \mathbb{R}_{+} \rightarrow \mathbb{R}_{+}$with $c(\beta) \downarrow 0$ when $\beta \downarrow 0$ such that, if $x \in \bar{B}(\hat{x}, \beta), x^{\prime} \in \bar{B}(\hat{x}, \beta)$ and $\varepsilon \in B\left(\varepsilon_{0}, \beta\right)$, then

$$
\left\|F\left(x^{\prime}, \varepsilon\right)-F(x, \varepsilon)-A\left(x^{\prime}-x\right)\right\| \leq c(\beta)\left\|x^{\prime}-x\right\| .
$$

Then there exists $\eta>0$ such that, for all $(x, \varepsilon)$ close enough to $\left(\hat{x}, \varepsilon_{0}\right)$, there exists $\bar{x}$ such that $F(\bar{x}, \varepsilon)=0$ and

$$
\|\bar{x}-x\| \leq \eta\|F(x, \varepsilon)\| .
$$

Proof. By the open mapping theorem, there exists a bounded right inverse of $A$, which we denote by $B$, i.e. a (possibly nonlinear) mapping $B: Y \rightarrow X$ such that $A B y=y$ for all $y \in Y$, and

$$
\|B\|:=\sup \{\|B y\|:\|y\| \mid y \in Y, y \neq 0\}
$$

is finite. Fix $\beta>0$ such that

$$
L_{\beta}:=c(\beta)\|B\|<1 .
$$

Let $\rho_{0}>0$ and take $x \in B\left(\hat{x}, \rho_{0}\right), \varepsilon \in \bar{B}\left(\varepsilon_{0}, \rho_{0}\right)$. By taking $\rho_{0}>0$ small enough, as $F$ is continuous, we may assume that

$$
\rho_{0}+\left(1-L_{\beta}\right)^{-1}\|B[F(x, \varepsilon)]\| \leq \beta .
$$

Let $\left\{x_{n}\right\}, n \in \mathbb{N}$, be the sequence defined by $x_{0}=x$ and the (modified Newton like) step

$$
x_{n+1}=x_{n}-B F\left(x_{n}, \varepsilon\right) .
$$

Then

$$
\left\|x_{n+1}-x_{n}\right\|=\left\|B\left[F\left(x_{n}, \varepsilon\right)\right]\right\| \leq\|B\|\left\|F\left(x_{n}, \varepsilon\right)\right\| .
$$

Relation (78) implies

$$
F\left(x_{n}, \varepsilon\right)+A\left(x_{n+1}-x_{n}\right)=0 .
$$


We show by induction that $\left\{x_{n}\right\}$ remains in $\bar{B}(\hat{x}, \beta)$. By $(77)$, this is true if $n=0$. For $n=1$, we have with (79) and (77)

$$
\left\|x_{1}-\hat{x}\right\| \leq\left\|x_{1}-x_{0}\right\|+\left\|x_{0}-\hat{x}\right\| \leq\left\|B\left[F\left(x_{0}, \varepsilon\right)\right]\right\|+\rho_{0} \leq \beta .
$$

Then if $x_{i} \in \bar{B}(\hat{x}, \beta)$, for all $1 \leq i \leq n,(73)$ and (80) imply

$$
\left\|F\left(x_{n}, \varepsilon\right)\right\| \leq c(\beta)\left\|x_{n}-x_{n-1}\right\| .
$$

Combining with (79), we get

$$
\left\|x_{n+1}-x_{n}\right\| \leq L_{\beta}\left\|x_{n}-x_{n-1}\right\| \leq \cdots \leq\left(L_{\beta}\right)^{n}\left\|x_{1}-x_{0}\right\|,
$$

and hence, with (77),

$$
\left\|x_{n+1}-x_{0}\right\| \leq\left(1-L_{\beta}\right)^{-1}\left\|x_{1}-x_{0}\right\| \leq\left(1-L_{\beta}\right)^{-1}\left\|B\left[F\left(x_{0}, \varepsilon\right)\right]\right\| \leq \beta-\rho_{0} .
$$

Since $\left\|x_{0}-\hat{x}\right\|<\rho_{0}$, we deduce that $x_{n+1} \in B(\hat{x}, \beta)$, and hence, the sequence $\left\{x_{n}\right\}$ remains in $B(\hat{x}, \beta)$. With (81) and (82), we obtain that $x_{n}$ converges to some $\bar{x}$ such that $F(\bar{x}, \varepsilon)=0$ and $\left\|\bar{x}-x_{0}\right\| \leq\left(1-L_{\beta}\right)^{-1}\|B\|\left\|F\left(x_{0}, \varepsilon\right)\right\|$, which proves (74) with constant $\eta$ given by

$$
\eta=\left(1-L_{\beta}\right)^{-1}\|B\|
$$

Remark. Note that, in the above proof, we have obtained the estimate (83) for the constant $\eta$ in (74), where $B$ is a bounded right inverse of $A$ and $L_{\beta}=$ $c(\beta)\|B\|$. Also, the hypothesis that $(x, \varepsilon)$ is "close enough" is satisfied whenever $x \in B\left(\hat{x}, \rho_{0}\right)$ and $\varepsilon \in \bar{B}\left(\varepsilon_{0}, \rho_{0}\right)$ where $\rho_{0}$ is such that (77) holds.

Corollary 21. Let the hypotheses of Theorem 20 hold, and denote by $B$ a bounded right inverse of $A$. Then for $\varepsilon$ close to $\varepsilon_{0}, F(\cdot, \varepsilon)$ has, in a neighborhood of $\hat{x}$, a zero denoted by $x_{\varepsilon}$ such that

$$
x_{\varepsilon}=\hat{x}-B F(\hat{x}, \varepsilon)+r(\varepsilon),
$$

where the remainder $r(\varepsilon)$ satisfies

$$
\|r(\varepsilon)\| \leq c(\beta)(1-c(\beta)\|B\|)^{-1}\|B\|^{2}\|F(\hat{x}, \varepsilon)\| .
$$

for some $\beta>0$ small enough.

Proof. Let $\hat{x}(\varepsilon):=\hat{x}-B F(\hat{x}, \varepsilon)$. We have that $F(\hat{x}, \varepsilon)+A(\hat{x}(\varepsilon)-\hat{x})=0$ and $\|\hat{x}(\varepsilon)-\hat{x}\| \leq\|B\|\|F(\hat{x}, \varepsilon)\|$. In view of $(73),\|F(\hat{x}(\varepsilon), \varepsilon)\| \leq c(\beta)\|B\|\|F(\hat{x}, \varepsilon)\|$. We conclude with the theorem.

Remark. According to (85), for the applications of Corollary 21, better estimates on $c(\beta)$ yield sharper bounds on the remainder in (84). The best constant $c(\beta)$ is given by

$$
c(\beta):=\sup _{x, x^{\prime}} \frac{\left\|F\left(x^{\prime}, \varepsilon\right)-F(x, \varepsilon)-A\left(x^{\prime}-x\right)\right\|}{\left\|x^{\prime}-x\right\|},
$$

where the supremum is taken over all $x \neq x^{\prime}$, both in $\bar{B}(\hat{x}, \beta)$. 


\section{References}

[1] F. Alvarez, J.F. Bonnans, and J. Laurent-Varin. Asymptotic expansion of the optimal control under logarithmic penalty: worked example and open problems. INRIA Report RR-6170, 2007.

[2] N. Bérend, J.F. Bonnans, J. Laurent-Varin, M. Haddou, and C. Talbot. Fast linear algebra for multiarc trajectory optimization. In G. Di Pillo and M. Roma, editors, Large scale nonlinear optimization, volume 83 of Nonconvex Optimization and Its Applications, pages 1-14. Springer, 2006.

[3] N. Bérend, J.F. Bonnans, J. Laurent-Varin, M. Haddou, and C. Talbot. An interior-point approach to trajectory optimization. AIAA J. of Guidance, Control and Dynamics, 30(5):1228-1238, 2007.

[4] M. Bergounioux, M. Haddou, M. Hintermüller, and K. Kunisch. A comparison of a Moreau-Yosida-based active set strategy and interior point methods for constrained optimal control problems. SIAM Journal on Optimization, 11:495-521 (electronic), 2000.

[5] J.T. Betts, S.K. Eldersveld, P.D. Frank, and J.G. Lewis. An interior-point algorithm for large scale optimization. In Large-scale PDE-constrained optimization (Santa Fe, NM, 2001), volume 30 of Lect. Notes Comput. Sci. Eng., pages 184-198. Springer, Berlin, 2003.

[6] J.F. Bonnans, J. Ch. Gilbert, C. Lemaréchal, and C. Sagastizábal. Numerical Optimization: theoretical and numerical aspects. Universitext. SpringerVerlag, Berlin, 2006. second edition.

[7] J.F. Bonnans and Th. Guilbaud. Using logarithmic penalties in the shooting algorithm for optimal control problems. Optimal Control, Applications and Methods, 24:257-278, 2003.

[8] J.F. Bonnans and J. Laurent-Varin. Computation of order conditions for symplectic partitioned Runge-Kutta schemes with application to optimal control. Numerische Mathematik, 103(1):1-10, 2006.

[9] A. Forsgren, P.E. Gill, and M.H. Wright. Interior methods for nonlinear optimization. SIAM Review, 44:525-597 (electronic) (2003), 2002.

[10] Clovis C. Gonzaga. Path-following methods for linear programming. SIAM Rev., 34(2):167-224, 1992.

[11] T. Jockenhövel, L. T. Biegler, and A. Wächter. Dynamic optimization of the tennessee eastman process using the optcontrolcentre. Computers and Chemical Engineering, 27:1513-1531, 2003.

[12] F. Leibfritz and E.W. Sachs. Inexact SQP interior point methods and large scale optimal control problems. SIAM Journal on Control and Optimization, 38:272-293 (electronic), 1999.

[13] Y. Nesterov and A. Nemirovskii. Interior-point polynomial algorithms in convex programming, volume 13 of SIAM Studies in Applied Mathematics. Society for Industrial and Applied Mathematics (SIAM), Philadelphia, PA, 1994. 
[14] A. Schiela and M.Weiser. Superlinear convergence of the control reduced interior point method for PDE constrained optimization. Comp.Opt.Appl., 39 (3):369-393, 2008.

[15] M. Ulbrich and S. Ulbrich. Primal-Dual Interior point methods for PDEconstrained optimization. Math. Program., 117:435-485, 2009.

[16] M. Weiser. Interior point methods in function space. SIAM J. on Control and Optimization, 44:1766-1786 (electronic), 2005.

[17] M. Weiser, T. Gänzler, and A. Schiela. A control reduced primal interior point method for a class of control constrained optimal control problems. Comp.Opt.Appl., 41 (1):127-145, 2008.

[18] M. Weiser and A. Schiela. Function space interior point methods for PDE constrained optimization. PAMM, 4 (1):43-46, 2004.

[19] S.J. Wright. Interior point methods for optimal control of discrete time systems. Journal of Optimization Theory and Applications, 77:161-187, 1993.

[20] S.J. Wright. Primal-dual interior-point methods. Society for Industrial and Applied Mathematics (SIAM), Philadelphia, PA, 1997. 


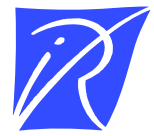

\section{Centre de recherche INRIA Saclay - Île-de-France Parc Orsay Université - ZAC des Vignes 4, rue Jacques Monod - 91893 Orsay Cedex (France)}

Centre de recherche INRIA Bordeaux - Sud Ouest : Domaine Universitaire - 351, cours de la Libération - 33405 Talence Cedex Centre de recherche INRIA Grenoble - Rhône-Alpes : 655, avenue de l'Europe - 38334 Montbonnot Saint-Ismier

Centre de recherche INRIA Lille - Nord Europe : Parc Scientifique de la Haute Borne - 40, avenue Halley - 59650 Villeneuve d'Ascq

Centre de recherche INRIA Nancy - Grand Est : LORIA, Technopôle de Nancy-Brabois - Campus scientifique 615, rue du Jardin Botanique - BP 101 - 54602 Villers-lès-Nancy Cedex

Centre de recherche INRIA Paris - Rocquencourt : Domaine de Voluceau - Rocquencourt - BP 105 - 78153 Le Chesnay Cedex

Centre de recherche INRIA Rennes - Bretagne Atlantique : IRISA, Campus universitaire de Beaulieu - 35042 Rennes Cedex

Centre de recherche INRIA Sophia Antipolis - Méditerranée : 2004, route des Lucioles - BP 93 - 06902 Sophia Antipolis Cedex 